\title{
Comparison of Convective Boundary Layer Velocity Spectra Retrieved from Large- Eddy-Simulation and Weather Research and Forecasting Model Data
}

\author{
Jeremy A. GibBS AND Evgeni Fedorovich \\ School of Meteorology, University of Oklahoma, Norman, Oklahoma
}

(Manuscript received 23 January 2013, in final form 24 July 2013)

\begin{abstract}
As computing capabilities expand, operational and research environments are moving toward the use of finescale atmospheric numerical models. These models are attractive for users who seek an accurate description of small-scale turbulent motions. One such numerical tool is the Weather Research and Forecasting (WRF) model, which has been extensively used in synoptic-scale and mesoscale studies. As finer-resolution simulations become more desirable, it remains a question whether the model features originally designed for the simulation of larger-scale atmospheric flows will translate to adequate reproductions of small-scale motions. In this study, turbulent flow in the dry atmospheric convective boundary layer (CBL) is simulated using a conventional large-eddy-simulation (LES) code and the WRF model applied in an LES mode. The two simulation configurations use almost identical numerical grids and are initialized with the same idealized vertical profiles of wind velocity, temperature, and moisture. The respective CBL forcings are set equal and held constant. The effects of the CBL wind shear and of the varying grid spacings are investigated. Horizontal slices of velocity fields are analyzed to enable a comparison of CBL flow patterns obtained with each simulation method. Two-dimensional velocity spectra are used to characterize the planar turbulence structure. One-dimensional velocity spectra are also calculated. Results show that the WRF model tends to attribute slightly more energy to larger-scale flow structures as compared with the CBL structures reproduced by the conventional LES. Consequently, the WRF model reproduces relatively less spatial variability of the velocity fields. Spectra from the WRF model also feature narrower inertial spectral subranges and indicate enhanced damping of turbulence on small scales.
\end{abstract}

\section{Introduction}

The Weather Research and Forecasting (WRF) model (Skamarock et al. 2008) has evolved toward a selfcontained numerical weather prediction system, capable of modeling atmospheric motions down to microscales. The promise of such capability is appealing to both operational and research environments where the accurate description of small-scale turbulent motions is increasingly desirable. Examples include air pollution applications, wave propagation studies, investigation of urban effects, and other general areas involving boundary layer dynamics. However, the ability of the WRF model to adequately capture the structure of atmospheric motions in the range of scales of the order of $100 \mathrm{~m}$ and smaller remains unclear.

Corresponding author address: Jeremy A. Gibbs, School of Meteorology, University of Oklahoma, 120 David L. Boren Blvd., Ste. 5900, Norman, OK 73072.

E-mail: gibbz@ou.edu
In this study, turbulent flow in the atmospheric convective boundary layer (CBL) is reproduced by the WRF model applied in a large-eddy-simulation (LES) mode (WRF-LES). Results are compared against those from a traditional LES code from the University of Oklahoma (OU-LES). To distill the fundamental differences between the results of each code, comparisons are performed within an idealized framework with realistic atmospheric conditions. The simulations use almost identical numerical grids and are initialized with the same vertical profiles of wind, temperature, and moisture. The respective CBL forcings were set equal and held constant across the entire 12-h simulation. The influence of wind shear (sheared CBL versus shear-free $\mathrm{CBL}$ ) and the effects of varying the numerical grid spacing $(20,40$, and $80 \mathrm{~m})$ were investigated.

Description of simulation setups and an overview of the evaluated flow statistics are presented in section 2 . In section 3, horizontal slices of simulated velocity fields are shown to enable comparison of CBL flow patterns 
obtained with each simulation method. Various traditional turbulence statistics are presented and analyzed in order to examine the simulated flow sensitivities to numerics employed by each simulation approach. One- and two-dimensional velocity spectra are demonstrated and evaluated as a means of gaining a better understanding of how turbulence is reproduced by OU-LES and WRFLES. The effects of spectral differences are illustrated through bulk CBL properties. The implications of these results are discussed in section 4.

\section{Experimental design}

\section{a. Model description and simulation setup}

The OU-LES code employs the Navier-Stokes equations of motion and the thermal energy equation in the Boussinesq approximation. The equations are integrated in time using a third-order Runge-Kutta scheme (Sullivan et al. 1996), with advection/convection terms being approximated using a second-order, centered, finitedifference scheme. Subgrid turbulence kinetic energy (STKE) closure is implemented after Deardorff (1980).

The core of OU-LES is derived from the Delft University LES (Nieuwstadt 1990), known to be a mature and established code. Changes were made in subsequent years to modernize the code, ranging in scope from minor (adding new postprocessing procedures) to major (upgrading the time-stepping scheme). Comparisons with wind tunnel measurements demonstrated that OU-LES is capable of reproducing sheared CBL flows (Fedorovich et al. 2001). Similarly, comparisons with bulk models and water tank data confirmed that OU-LES is able to reproduce turbulent flow regimes associated with free convection in the atmospheric boundary layer (Fedorovich et al. 2004a). In addition, OU-LES was compared with five other representative contemporary LES codes (Fedorovich et al. 2004b) and performed consistently with these codes in the reproduction of different flow turbulence statistics and across a variety of CBL cases (sheared and shear free).

The WRF-LES solves the fully compressible NavierStokes equations in the flux form. Because of compressibility, acoustic and other high-frequency computational modes arise in the simulations. To maintain stability while keeping a feasible integration time step, the equations are integrated in time using a time-split version of the thirdorder Runge-Kutta scheme (Wicker and Skamarock 1998, 2002). This procedure introduces three additional filters in order to maintain the numerical stability of the code: three-dimensional divergence damping, vertically integrated horizontal divergence damping, and semi-implicit acoustic step off-centering (Skamarock et al. 2008).
Horizontal advection is accomplished with a fifth-order, upwind-biased, finite-difference scheme, while vertical advection is carried out by means of a third-order scheme. The WRF-LES employs a nearly identical Deardorff STKE scheme to that of OU-LES.

The coefficients that determine the performance of each time-splitting filter are run-time adjustable. As will be discussed in section $3 \mathrm{c}$, the modification or disengagement of these filters is not advisable (J. Dudhia 2013, personal communication). The order of advection approximation is also run-time configurable, ranging from second to sixth order. The decision to employ a particular advection scheme in this study was based on practical considerations. First, the fifth-order upwind scheme is recommended to WRF model users because it reduces phase propagation errors as compared to lower-order schemes (Klemp and Skamarock 2004). This particular advection scheme was also found to perform the best when employed in combination with the third-order Runge-Kutta time-integration scheme (Wicker and Skamarock 2002). These characteristics are especially appealing to those users who are interested in the simulation of realistic atmospheric environments. Second, the WRF model package incorporates an idealized CBL case designed to introduce users to the model's LES capabilities. The default settings for this case employ a fifth-order advection scheme. Even for experienced users, this option might appear as the recommended one. The above considerations determined the choice of this scheme for the reported study. Based on similar considerations, the Deardorff (1980) 1.5-order prognostic TKE model was chosen as the WRF-LES subgrid closure scheme.

Identical $10.24 \times 10.24 \times 2 \mathrm{~km}^{3}$ numerical domains were used for runs with both codes. Isotropic grid spacing was used, with $\Delta x=\Delta y=\Delta z$ being varied among 20, 40, and $80 \mathrm{~m}$. At the lower boundary, a roughness of $14 \mathrm{~cm}$ was employed and Monin-Obukhov flux-profile relationships (Monin and Obukhov 1954; Dyer and Hicks 1970) were used as boundary conditions. Both codes applied the same Rayleigh damping in the upper portion of the simulation domain. Lateral boundary conditions were periodic. WRF-LES and OU-LES runs were initialized with the same idealized profiles of temperature and moisture depicted in Fig. 1. These profiles are packaged with the WRF model's idealized LES test case. In addition to the varying grid spacing, differing CBL flow types (with and without mean wind) were investigated. The shear-free case was initialized with zero wind, while the shear-driven case was initialized with a spatially uniform, geostrophically balanced $x$-component wind velocity $u$ of $10 \mathrm{~ms}^{-1}$. Surface kinematic heat and moisture fluxes in all simulated cases were set equal to 

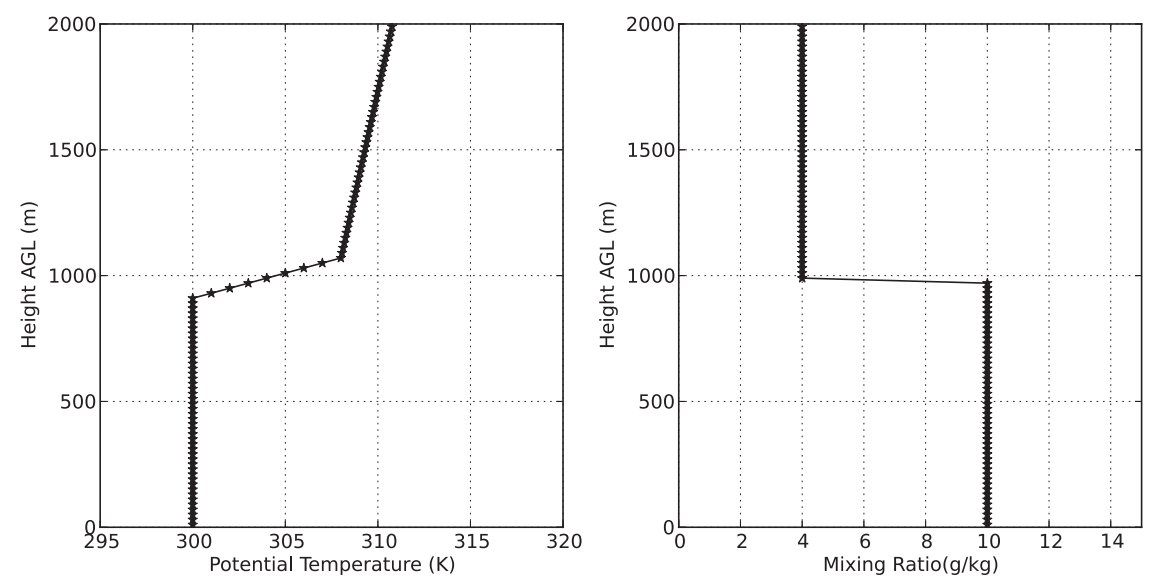

FIG. 1. Idealized profiles of temperature and moisture used to initialize all simulations.

$0.12 \mathrm{~K} \mathrm{~m} \mathrm{~s}^{-1}$ and $5 \times 10^{-5} \mathrm{~m} \mathrm{~s}^{-1}$, respectively, and held constant during the entire 12-h simulation period.

\section{b. Evaluated flow statistics}

Horizontal slices of simulated velocity fields were taken at different elevations above the ground. In our further analysis, we focus on the velocity data referring to the level of $z / z_{i}=0.25$, where $z$ is the height above ground level (AGL) and $z_{i}$ is the depth of the boundary layer evaluated from the horizontally averaged profiles of the vertical kinematic heat flux. This height was chosen in order to eliminate direct effects of the surface on the flow field while remaining in the bulk of the simulated CBL.

Slices of horizontal $\left(u^{\prime}, v^{\prime}\right)$ and vertical $\left(w^{\prime}\right)$ velocitycomponent perturbations (which are deviations from the plane means, $u, v$, and $w$, respectively) were captured at every minute within each code's final simulation hour. The series of these turbulent perturbations were obtained by sampling data across the entire horizontal plane. Vertical profiles of normalized velocity variances $\left.\left\langle\overline{u^{\prime 2}}\right\rangle / w_{*}^{2}, \overline{\left\langle v^{\prime 2}\right.}\right\rangle / w_{*}^{2}$, and $\left.\overline{\left\langle w^{\prime 2}\right.}\right\rangle / w_{*}^{2}$ were calculated in order to quantify the overall variability of the flow field. Here, the overbars signify the spatial (over the horizontal plane) averaging and the angle brackets denote temporal (over $1 \mathrm{~h}$ ) averaging. The quantity $w *=\left(B_{s} z_{i}\right)^{1 / 3}$ is the convective velocity scale, originally suggested by Deardorff (1970), with

$$
B_{s}=\frac{g}{\theta_{0}}\left\langle\overline{w^{\prime} \theta_{v}^{\prime}}\right\rangle_{s}
$$

being the near-surface (subscript $s$ ) value of the vertical turbulent buoyancy flux. In the above expression, $g$ is the acceleration due to gravity, $\theta_{0}$ is a constant potential temperature reference value, and $\left\langle\overline{{w^{\prime} \theta_{v}^{\prime}}_{v}}\right\rangle$ is the kinematic virtual heat flux $\left(\theta_{v}^{\prime}\right.$ is the virtual potential temperature perturbation). Normalized profiles of turbulence kinetic energy (TKE) $\left.0.5\left(\overline{\left\langle{u^{\prime 2}}^{2}\right.}+\overline{\left\langle v^{\prime 2}\right.}\right\rangle+\overline{\left\langle{w^{\prime 2}}^{2}\right.}\right) / w_{*}^{2}$ were also invoked for analysis. For the sheared CBL case, a normalized vertical distribution of kinematic vertical momentum flux $\left\langle\overline{w^{\prime} u^{\prime}}\right\rangle / w_{*}^{2}$ was used to quantify the vertical turbulent transport of momentum. For that case, we also looked at the normalized mean $u$-component profile $\langle\bar{u}\rangle / w *$.

The emphasis in the analysis was placed on the normalized one- and two-dimensional spectral density functions (spectra) that were employed to investigate the scale structure of simulated velocity fields. Onedimensional spectra of resolved velocity components were calculated in the $x$ and $y$ directions following the method described in Kaiser and Fedorovich (1998). Twodimensional resolved-velocity spectra were calculated by applying the planar Fourier transform as outlined in Kelly and Wyngaard (2006).

To better understand the turbulence properties exhibited through each of the two types of employed spectral techniques, we investigated velocity-component distributions, variances, turbulence kinetic energy, and turbulent momentum flux in conjunction with and complementary to spectra. The calculated statistics were used to gain insights into the ability of each code to reproduce turbulent motions in both the shear-free and sheared CBLs. Horizontal slices allowed quick visual inspection of the spatial structure of the velocity fields. However, as will be demonstrated in section 3, visual similarity of velocity slices can be misleading when surmising turbulence-resolving capabilities of a given code. Thus, exploiting turbulence statistics and spectra for the structural analyses of simulated CBL flows appears to be of vital importance.

As noted by Skamarock (2004), who used turbulence kinetic energy spectra to evaluate the performance of 
mesoscale numerical weather prediction models, investigation of velocity spectra proves to be an appealing analytical tool for several reasons (despite not being a mainstream model validation technique). First, there is a glaring lack of direct model verification data on the scales of motion that are of interest for this study (tens of meters in size and seconds in duration). Second, spectra can be indicative of a given simulation technique's ability to produce energy spectral distributions that are consistent with the state-of-the-art understanding of turbulence dynamics. Finally, the spectral analysis allows a detailed evaluation of the effects of model numerics on turbulent motions of different scales and the assessment of the effective model resolution.

\section{Results}

Statistics for simulated flow fields described in section 2 are presented below for both the shear-free and sheared CBL cases. The behavior of the statistics was generally consistent across disparate grid spacings. Accordingly, results are only shown for simulations with the finest grid spacing $(20 \mathrm{~m})$. All fields are shown for the final hour of simulation. Planar slices depict the instantaneous velocity fields at the final moment of the corresponding simulation described in section 2. Histograms represent the distributions of instantaneous velocity values over the horizontal plane throughout the last hour of simulation.

\section{a. Instantaneous resolved fields}

Instantaneous contours of the simulated $x$ component of the velocity $u$ perturbation in the horizontal plane are shown in Fig. 2. Velocity patterns for the shear-free case are demonstrated in Figs. 2a (OU-LES field) and 2b (WRF-LES field). Fields produced by both codes appear visually similar, depicting random, evenly distributed velocity fluctuations expected in the absence of a mean wind. Results from the shear-driven CBL case (Figs. 2c,d) point to the potentially overall stronger variability of the horizontal wind field as a result of the imposed mean shear. In visual terms, fields reproduced by both codes look to be similar at a cursory glance.

Instantaneous contours of the simulated $z$ component of velocity $w$ in the horizontal plane are shown in Fig. 3 . Values for the shear-free case are shown in Figs. 3a and 3b. As expected, both codes depict similar cell-type convective structures. At first glance, the fields appear to be nearly indistinguishable. Careful inspection, however, reveals the potential that WRF-LES may produce slightly more amorphous, less organized structures than OU-LES does. Results from the shear-driven CBL case, shown in Figs. $3 c$ and $3 d$, indicate that both codes successfully reproduce roll-like structures that are expected in a $\mathrm{CBL}$ with a strong wind shear (Moeng and Sullivan 1994). While patterns resulting from both codes again appear to be visually congruent, the WRF-LES field depicts slightly more organized, elongated streaks as compared with the structures in the OU-LES output.

Visual comparisons of simulated velocity patterns allow for quick inspection of model performance. Overall, the velocity contours in the discussed cases are so similar that they might suggest to users that the solutions only differ very slightly. In that sense, other techniques are needed to differentiate the solutions in a quantitative manner. Our goal would be to gain insights into the underlying turbulence dynamics in order to ascertain the origin and relative importance of the subtle differences in the velocity patterns. Such differences may be indicative of significant disparities between the two simulated flows in terms of turbulence dynamics, energetics, and scale interactions.

\section{b. One-dimensional spectra}

At each simulation output time (every minute within the last hour of simulation) one-dimensional velocitycomponent spectral densities (spectra) were calculated for every row in the direction of interest $(x$ or $y)$ and subsequently averaged over the corresponding orthogonal direction ( $y$ or $x$ ), thus providing 60 mean directional spectra. The average of these spectra provided the resultant one-dimensional spectrum in the $x$ or $y$ direction. In other words, the value at each wavenumber is obtained by averaging across a sample of $60 \times 512$ realizations. The 1-h averaging interval falls between the convective overturning time scale $(\approx 20 \mathrm{~min})$ and the semidiurnal time scale (approximately several hours).

For the $u$ component of velocity, $x$ is therefore the longitudinal direction, with the corresponding wavenumber defined as $k_{1}=2 \pi / \lambda_{x}$, and $y$ is the transverse direction, with the wavenumber defined as $k_{2}=2 \pi / \lambda_{y}$, where $\lambda_{x}$ and $\lambda_{y}$ are wavelengths along the $x$ and $y$ coordinates, respectively. Accordingly (Pope 2000), $P_{u}\left(k_{1}\right)$ denotes the one-dimensional longitudinal $u$-component velocity spectrum and $P_{u}\left(k_{2}\right)$ denotes the one-dimensional transverse $u$-component velocity spectrum. The calculated spectra of the $u$ component are illustrated in Figs. 4a and $4 \mathrm{~b}$ for the shear-free case and in Figs. $4 \mathrm{c}$ and $4 \mathrm{~d}$ for the sheared CBL case. Figures $4 \mathrm{a}$ and $4 \mathrm{c}$ represent normalized spectra calculated in the $x$ direction, $P_{u}\left(k_{1}\right)$, and Figs. $4 \mathrm{~b}$ and $4 \mathrm{~d}$ show the $y$-direction spectra $P_{u}\left(k_{2}\right)$. When analyzing differences of spectral densities between OULES and WRF-LES, one should keep in mind that the spectral plots are presented using logarithmic scales traditionally employed in the spectral analyses of atmospheric turbulence. While differences between the scaled spectra appear to be visually small, plotting those 
(a)

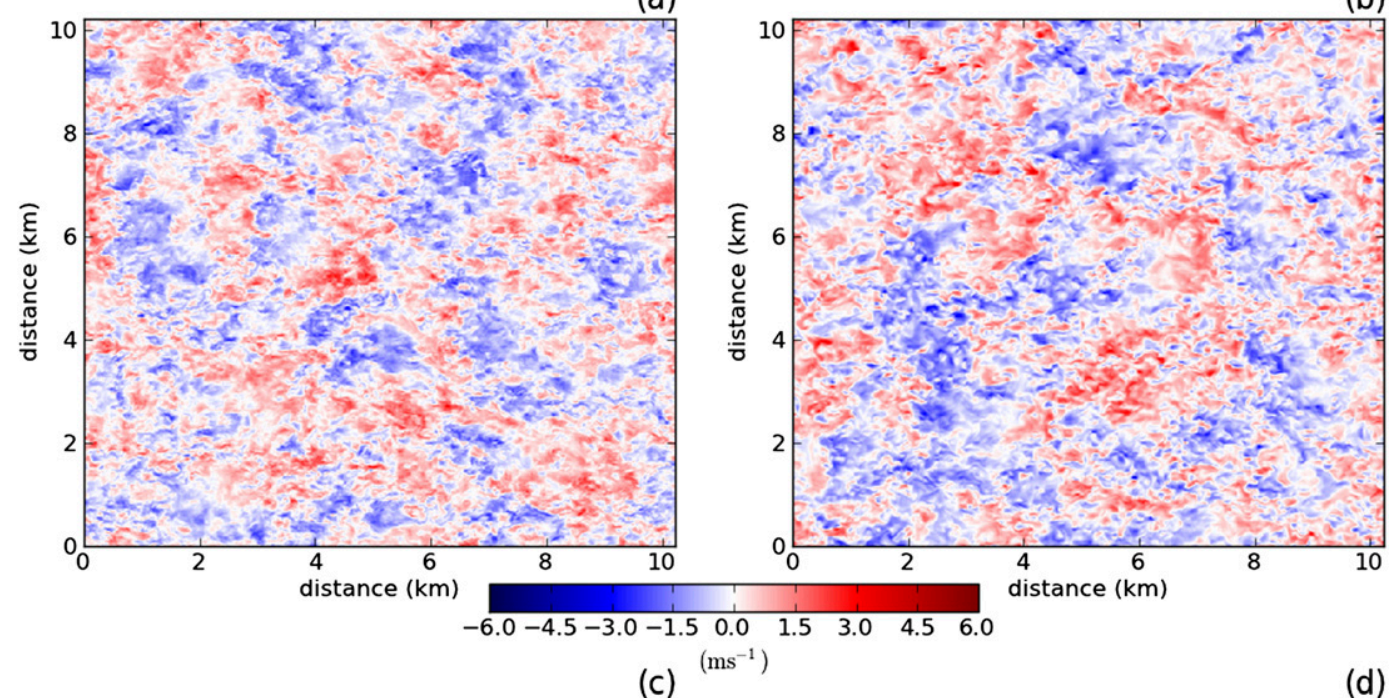

(b)

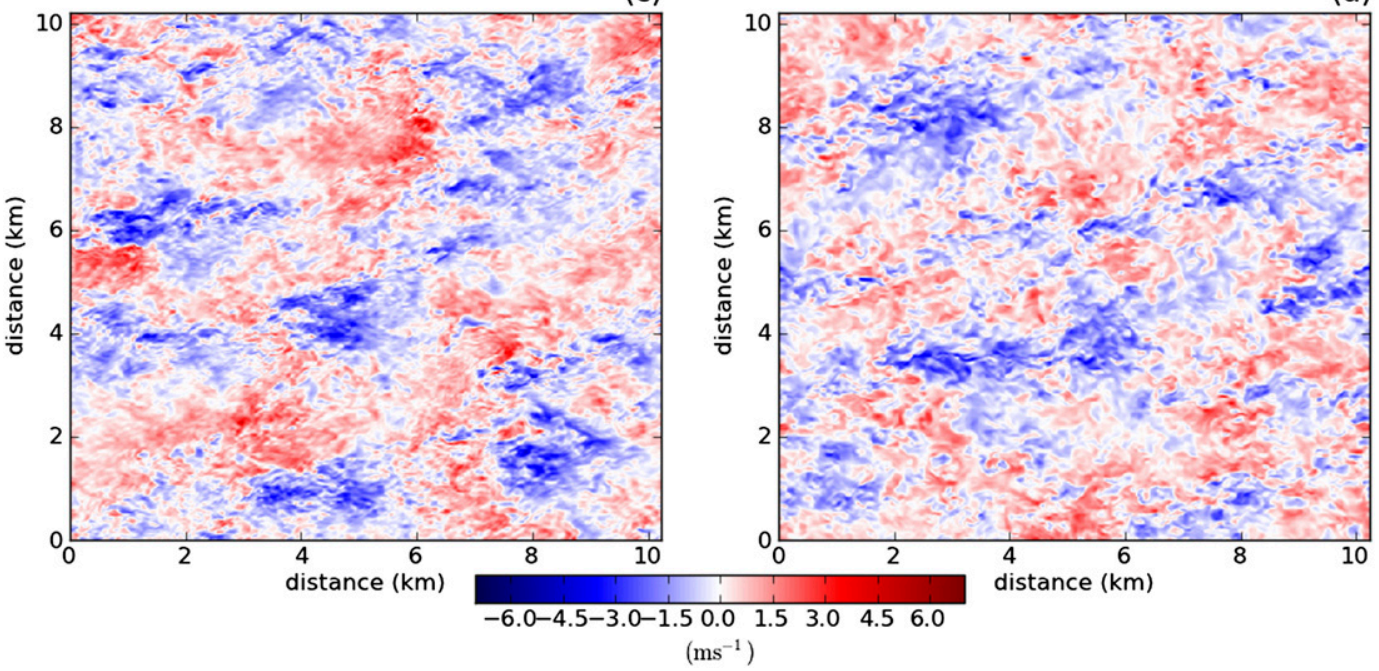

FIG. 2. Horizontal slice of the $u$-component velocity at level $z / z_{i}=0.25$ during the final hour of the simulation window in the (a),(b) shear-free and (c),(d) sheared CBL for the (left) OU-LES and (right) WRF-LES fields.

as fractional differences, the relative differences between the spectra appear to be significant (the corresponding figures are not shown). For example, the relative differences between the $u$-component OU-LES and WRF-LES spectra in the shear-free CBL approach $25 \%$ in the lower-wavenumber range.

Examination of $u$-component spectra in the shear-free case highlights key differences between the two considered simulation techniques in the representation of velocity spectra. First, one may notice that WRF-LES spectra show more energy in large-scale motions compared to OU-LES spectra. Second, energy drops off faster with decreasing scales of motion (larger wavenumbers) in the WRF-LES spectra than in the OU-LES spectra. The dropoff wavenumber in the WRF-LES spectra is generally consistent with its values discussed in Skamarock (2004), where the dropoff point was found to lie in the range from $5 \Delta h$ to $8 \Delta h$, where $\Delta h$ is the horizontal grid spacing. As a result, the WRF-LES spectra exhibit notably shorter inertial subranges than do the OU-LES spectra. One may also notice that the spectral behavior is quite similar for the longitudinal and transverse directions. Such behavior is expectable since there is no directional effect of mean shear on the simulated turbulence in this case.

In the sheared CBL case, a relatively fast dropoff with increasing wavenumber is observed in both the WRF-LES and OU-LES spectra as compared with their shear-free counterparts in Figs. $4 \mathrm{a}$ and $4 \mathrm{~b}$. The rate of decline in the WRF-LES spectra in this case matches closely that in the OU-LES spectra, although the spectral decay still happens faster in the WRF-LES spectra. The stronger 
(a)

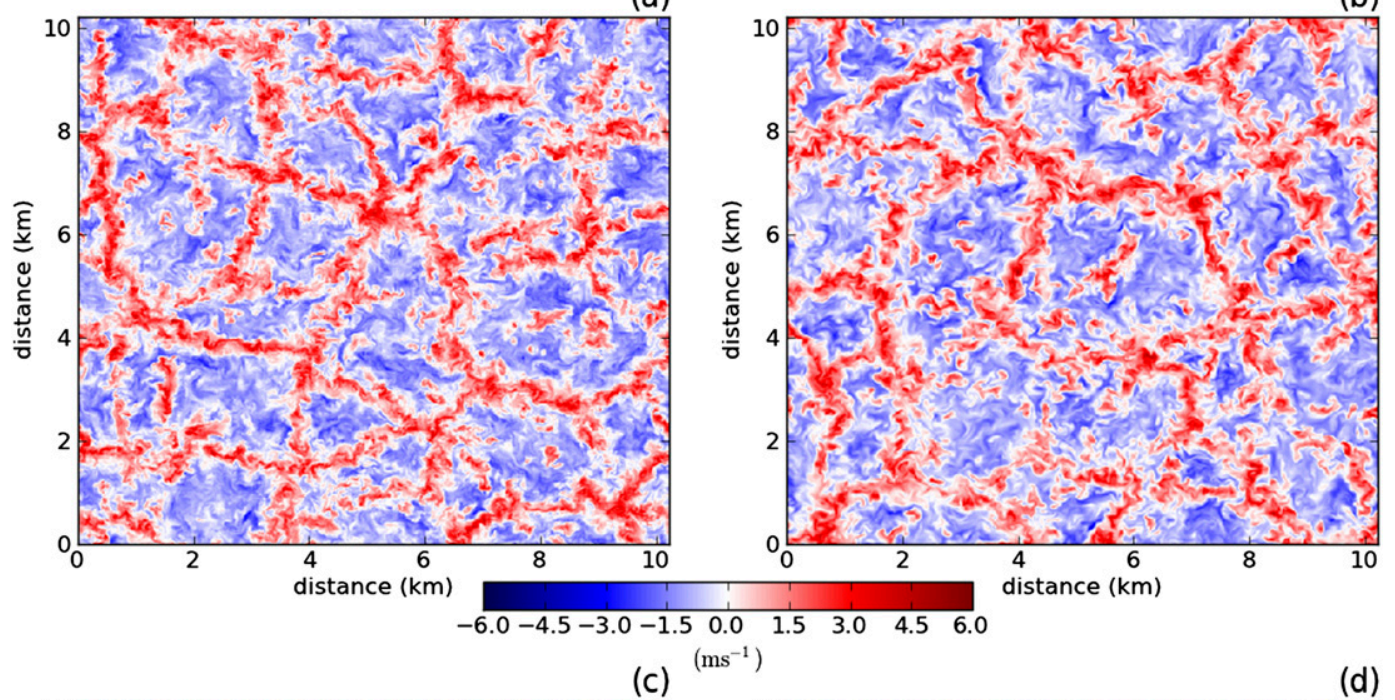

(b)

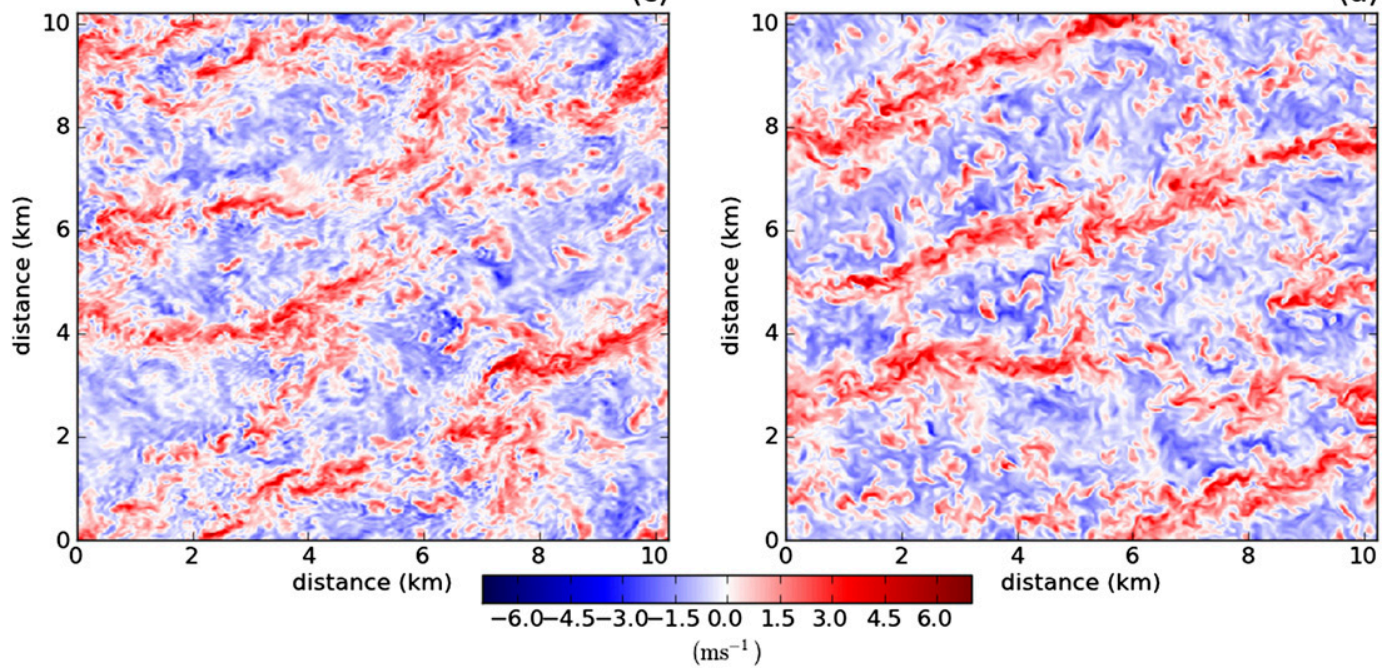

FIG. 3. As in Fig. 2, but for the $w$ component of the velocity.

damping of the small-scale variability of turbulent motions in the presence of mean shear is apparently a result of the smoothing effect of the time-stepping numerical scheme that is common for both codes. Note that for the WRF-LES spectrum, the dropoff point is closer to that in the longitudinal OU-LES spectrum (Fig. 4c) as compared with the transverse spectra (Fig. 4d). Since roll structures are primarily oriented in the along-wind $x$ direction, this means that WRF-LES produces relatively fewer small-scale variations in the cross-roll direction as compared with variations in the mean flow direction. We will try to explain later such extra damping of the smallscale motions in WRF-LES.

The normalized one-dimensional spectra of the vertical velocity component $w$ are shown in Fig. 5. Like in the case of the $u$-component spectra, Figs. $5 \mathrm{a}$ and $5 \mathrm{~b}$
(Figs. 5c,d) present data for shear-free (sheared) CBL. Figures 5a and 5c (Figs. 5b,d) display the normalized $P_{w}\left(k_{1}\right)\left[P_{w}\left(k_{2}\right)\right]$ spectra, which are all transverse spectra with respect to the $w$ field. From comparing the $w$-component spectra for the shear-free case, WRF-LES attributes slightly more energy to larger scales and predicts an earlier and faster turbulence damping on smaller scales in comparison with OU-LES. The inertial subranges with $-2 / 3$ spectral behavior in the $w$ spectra are noticeably broader than in the $u$ spectra (cf. Figs. $5 a, b$ and Figs. $4 a, b)$, mostly at the expense of the inertial intervals protruding farther into direction of larger scales. Within the main portion of the reproduced wavenumber interval, the OU-LES and WRF-LES spectra are in close agreement with each other. Spectral behavior is similar in both directions $\left(k_{1}\right.$ and $\left.k_{2}\right)$, as expected, since 


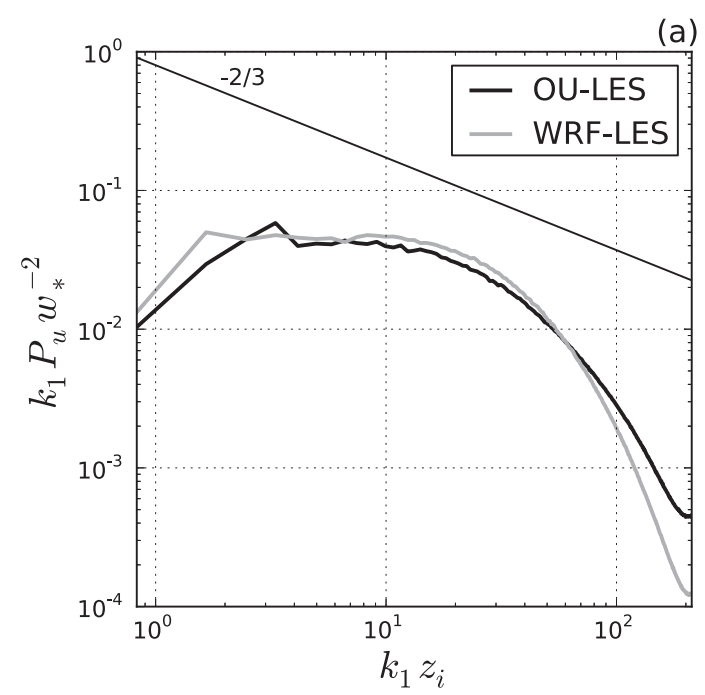

(a)
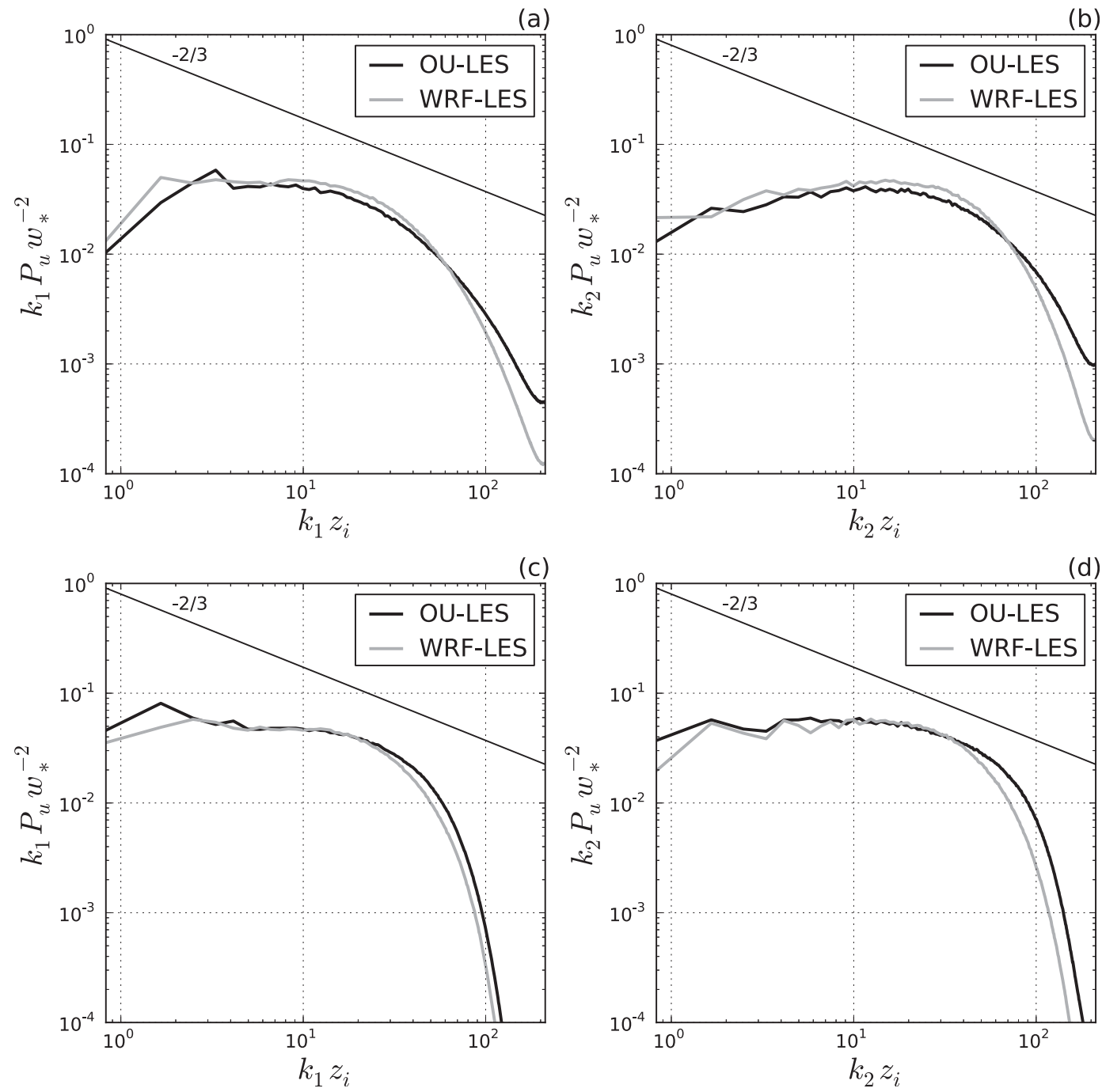

(c)

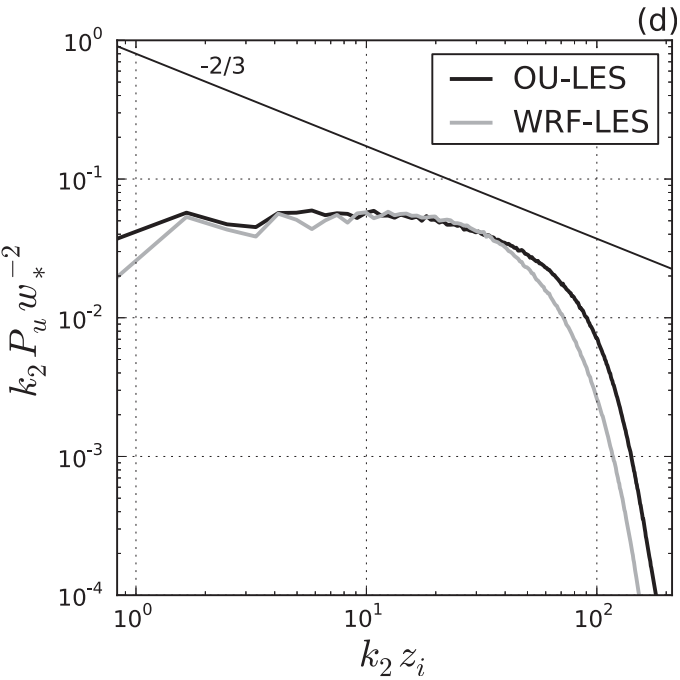

FIG. 4. Normalized one-dimensional spectral density (spectra) of the $u$ component of the velocity in the (a),(c) longitudinal $\left(k_{1}\right)$ and (b),(d) transverse $\left(k_{2}\right)$ directions at $z / z_{i}=0.25$ for the (top) shear-free and (bottom) sheared CBL cases. The straight lines indicate the $-2 / 3$ spectral behavior that is expected in the inertial subrange.

there are no shear-induced directional effects in place. Spectral curves from both codes are nearly identical in the along-roll direction (approximately along $x$ ) for the sheared CBL case, where WRF-LES and OU-LES predict normalized spectra to be rather flat within the production ranges (Fig. 5c). However, OU-LES predicts stronger spectral damping at the very large-scale spectral end than WRF-LES. The relative flatness of the production-range $w$ spectra in the sheared CBL has been previously pointed out in the wind-tunnel experiments of Kaiser and Fedorovich (1998). The observed disparity between $k_{1}$ and $k_{2}$ spectra (cf. Figs. $5 \mathrm{c}$ and $5 \mathrm{~d}$ ) is likely a result of the directional effect of the mean shear. Note in this connection a markedly wider inertial subrange in the $k_{2}$-direction spectrum (Fig. $5 \mathrm{~d}$ ), which is computed along the direction that is roughly transverse with respect to direction of the mean shear.

The initial implications that may be deduced from analyses of the one-dimensional velocity spectra are twofold. First, the WRF-LES spectra consistently decline from the theoretically expected spectral behavior earlier (at smaller wavenumber values) and this decline is faster than in the OU-LES spectra. This suggests that the spectral dynamics in WRF-LES is affected by some damping on smaller scales of motion and apparently results in a lower effective resolution of WRF-LES compared to the OU-LES code. The relative (in terms of grid-cell size factor) location of the deviation point in the WRF-LES spectra is consistent with results from previous studies [e.g., with those of Skamarock (2004)]. 

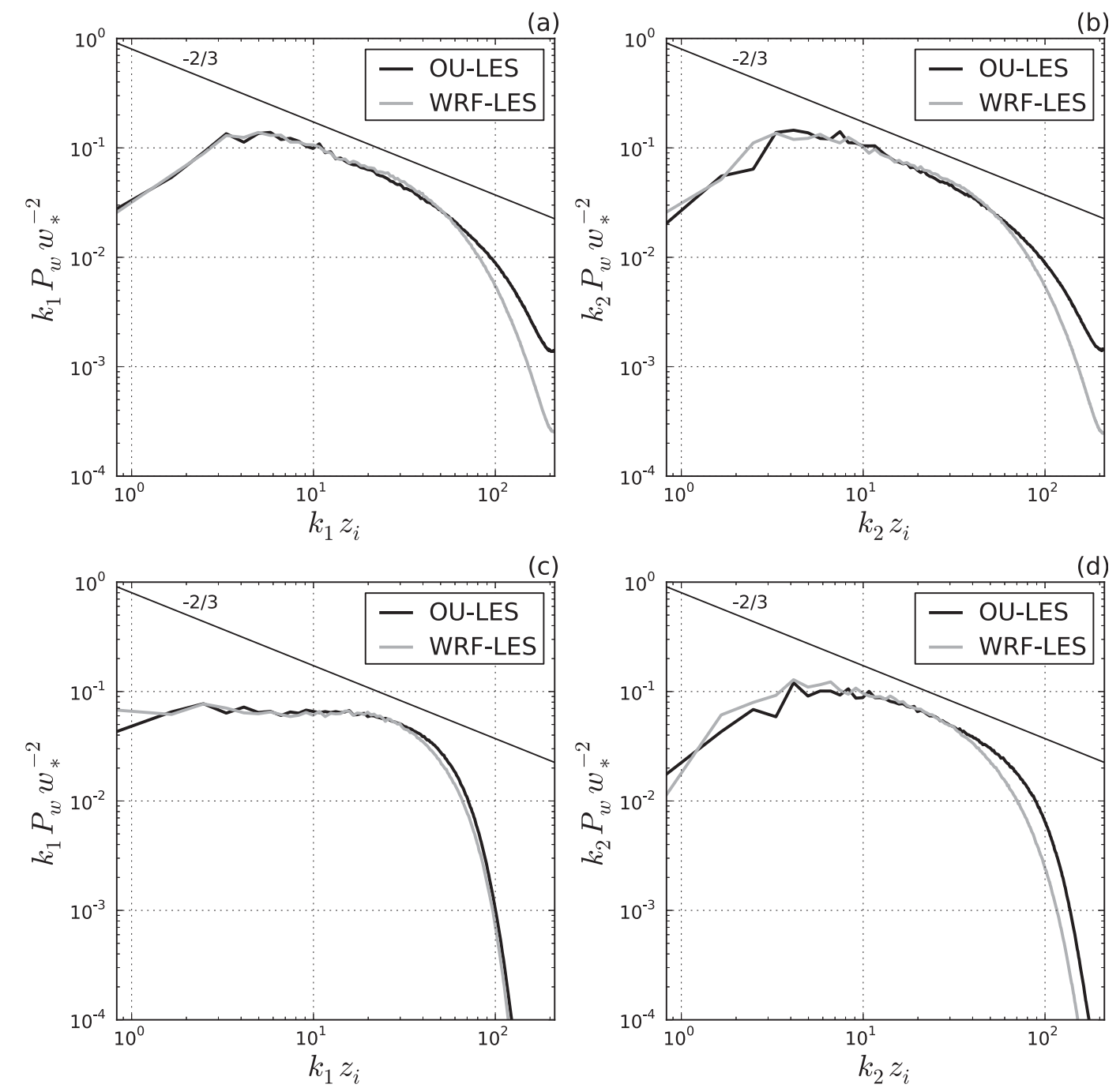

FIG. 5. As in Fig. 4, but for the $w$ component of velocity in the transverse (a),(c) $k_{1}$ and (b),(d) $k_{2}$ directions.

Second, the extent of the enhanced spectral damping is dependent on the mean shear effect in the CBL. Namely, the wavenumber range within which the WRFLES spectra deviate from both the theoretical spectral slope and the OU-LES spectra is different for the shearfree and sheared CBL cases. This lends credence to the idea that internal features of the WRF-model numerics are, at least partially, to blame for such spectral damping.

\section{c. Two-dimensional spectra}

Further insights into energy partitioning among different scales of motion and the role of the directional effect of shear in this partitioning may be gained from normalized two-dimensional (2D) spectral densities (2D spectra) of flow velocity components. Normalized 2D spectra of the $u$ component of velocity in the $(x, y)$ plane at $z / z_{i}=0.25$ are shown in Fig. 6. Physical coordinate directions $x$ and $y$ in the wavenumber space correspond to $k_{1}$ and $k_{2}$, respectively. The largest turbulence scales in the shown 2D spectra thus refer to the center of the spectral plot. The scales decrease outward (toward large $\left|k_{1}\right|$ and $\left.\left|k_{2}\right|\right)$, and the contour lines represent constant spectral density values. Figures $6 a$ and $6 b(6 c, d)$ correspond to the shear-free (sheared) CBL cases. Figures $6 \mathrm{a}$ and $6 c(6 b, d)$ depict spectra calculated from OU-LES (WRF-LES) outputs.

In the shear-free CBL case (Figs. 6a,b), the $u$ spectra are elongated in the transverse direction $\left(k_{2}\right)$. This is consistent with turbulence theory, which predicts that the ratio of longitudinal to transverse spectra in the inertial subrange should be larger than one (Tennekes and Lumley 1972). For isotropic turbulence, in particular, this ratio is equal to $4 / 3$. When compared with the $2 \mathrm{D}$ OU-LES spectra, the WRF-LES spectral densities look 
(a)
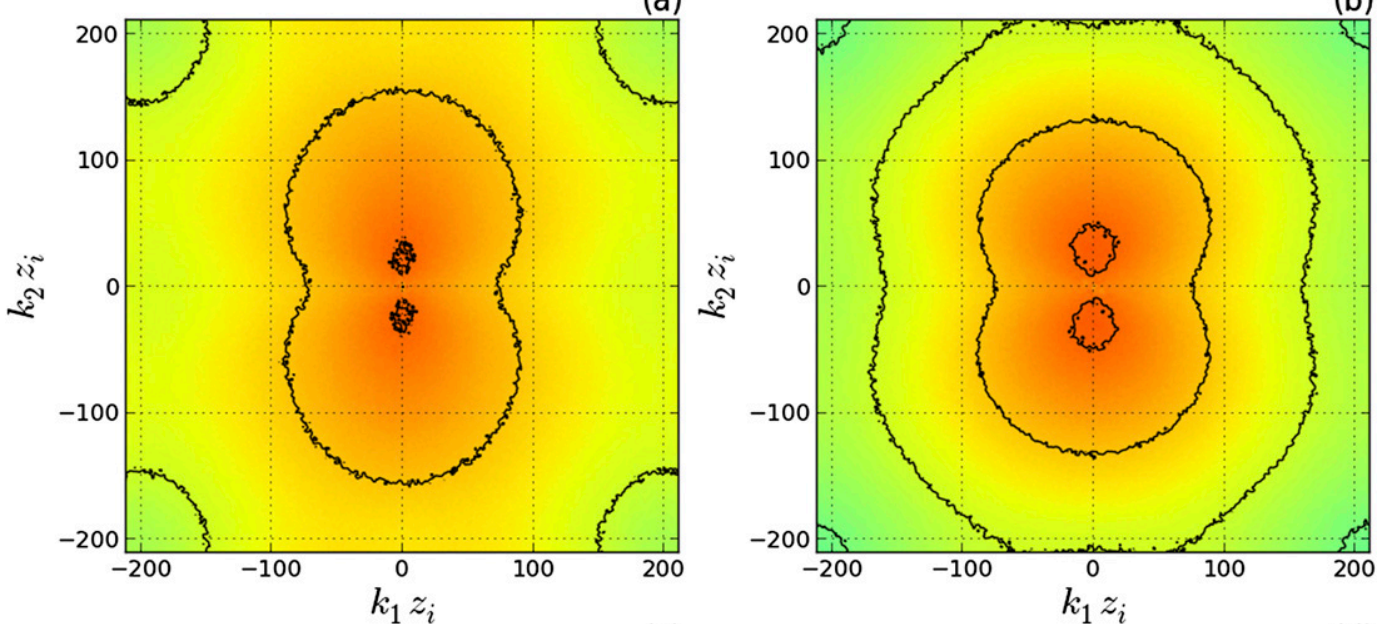

(c)

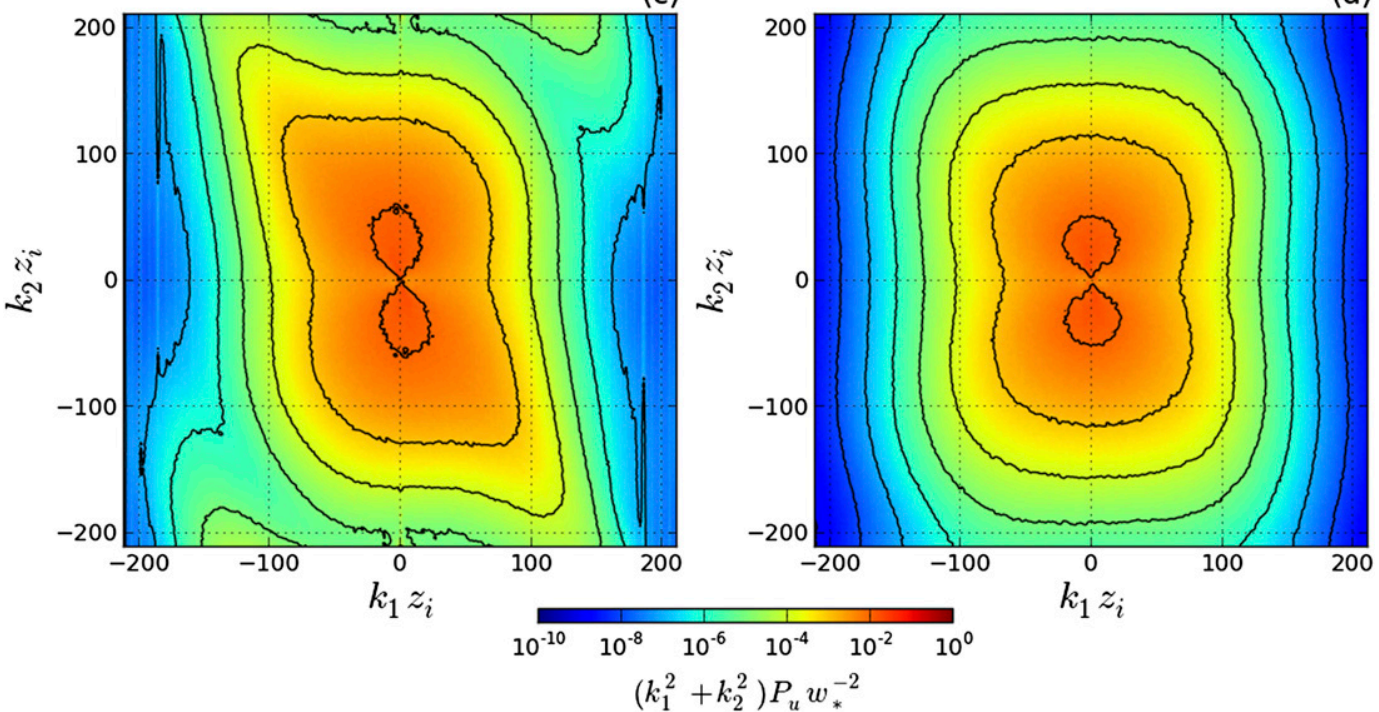

(d)

FIG. 6. Normalized two-dimensional spectra of the $u$ component of the velocity at $z / z_{i}=0.25$ for the (a),(b) shearfree and (c),(d) sheared CBL cases. The (left) OU-LES and (right) WRF-LES spectra are shown. Contours represent spectral density in powers of 10, decreasing outward from the center. The center contours are equal for both OU-LES and WRF-LES.

more compressed toward larger scales (small wavenumbers) with a sharp dropoff at large wavenumbers. This spectral behavior is consistent with that of the longitudinal and transverse one-dimensional spectra of $u$ in Fig. 4. In the sheared CBL (Figs. 6c,d), 2D spectrum from OU-LES output is starkly different from the WRFLES spectrum. The latter spectrum is again more compressed toward larger scales, although the effect is not as prominent as in the shear-free CBL case (Fig. 6b). Contours are elongated in the transverse directions, which is again consistent with turbulence theory predictions. Note, however, that in the OU-LES spectrum (Fig. 6c) the spectral density contours are stretched in the transverse direction at large $\left|k_{2}\right|$ values (corresponding to fine turbulence scales). This feature is not found in the WRF-LES spectrum. To interpret this particular smallscale spectral feature, consider the velocity field structure for this case (Fig. 3). This field is predominantly composed of roll-like structures that are primarily oriented in the along-wind direction. Hence, variations in velocity are expected to be smaller along the rolls as opposed to the cross-roll direction. The stretched portions in the OU-LES spectrum apparently depict these shear-induced velocity field features on small scales. These features are not present in the WRF-LES spectra. Such directionally dependent turbulence structure features in 2D velocity spectra for the sheared CBL match the features that were previously pointed out in the 
(a)
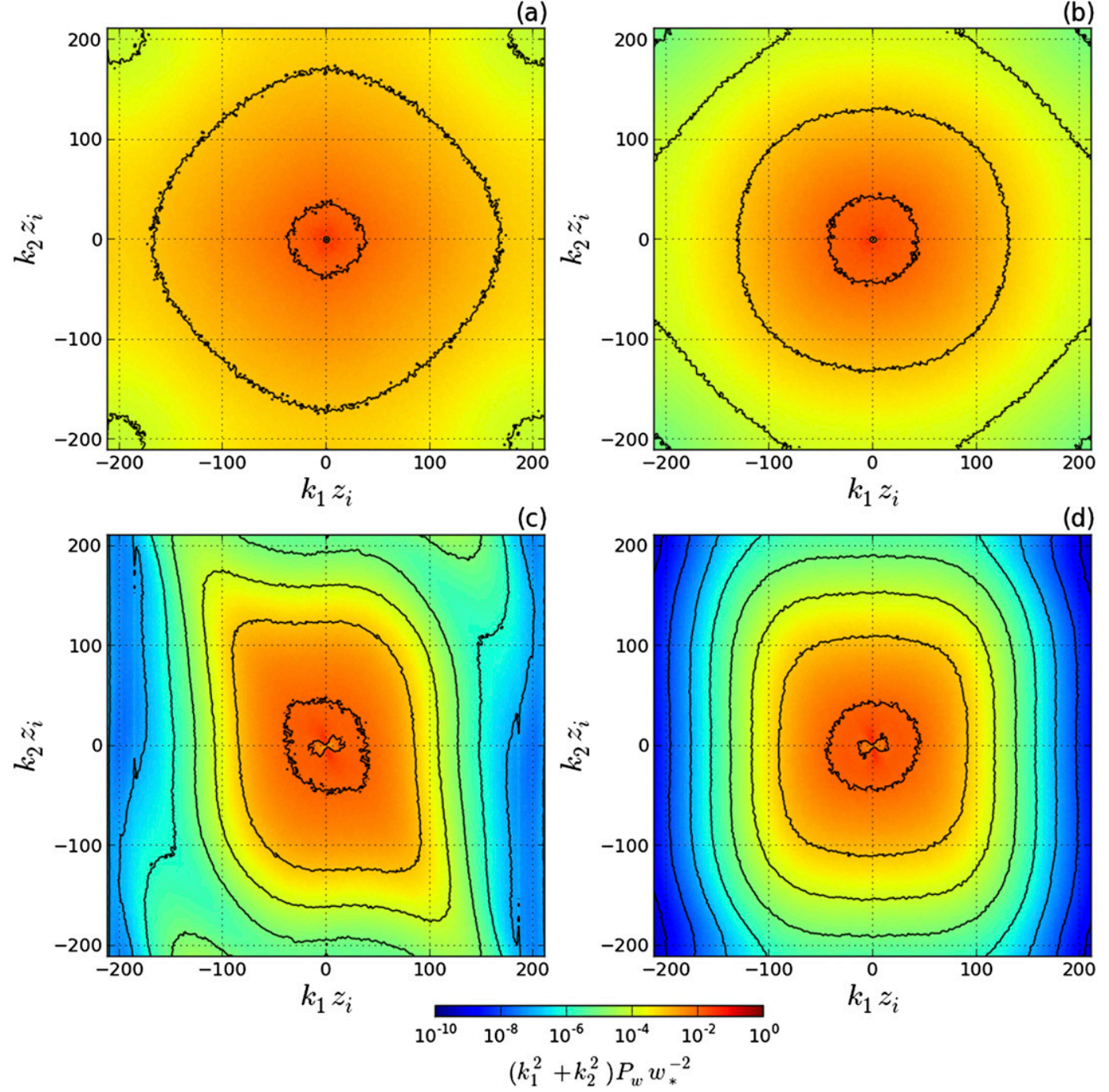

(c)

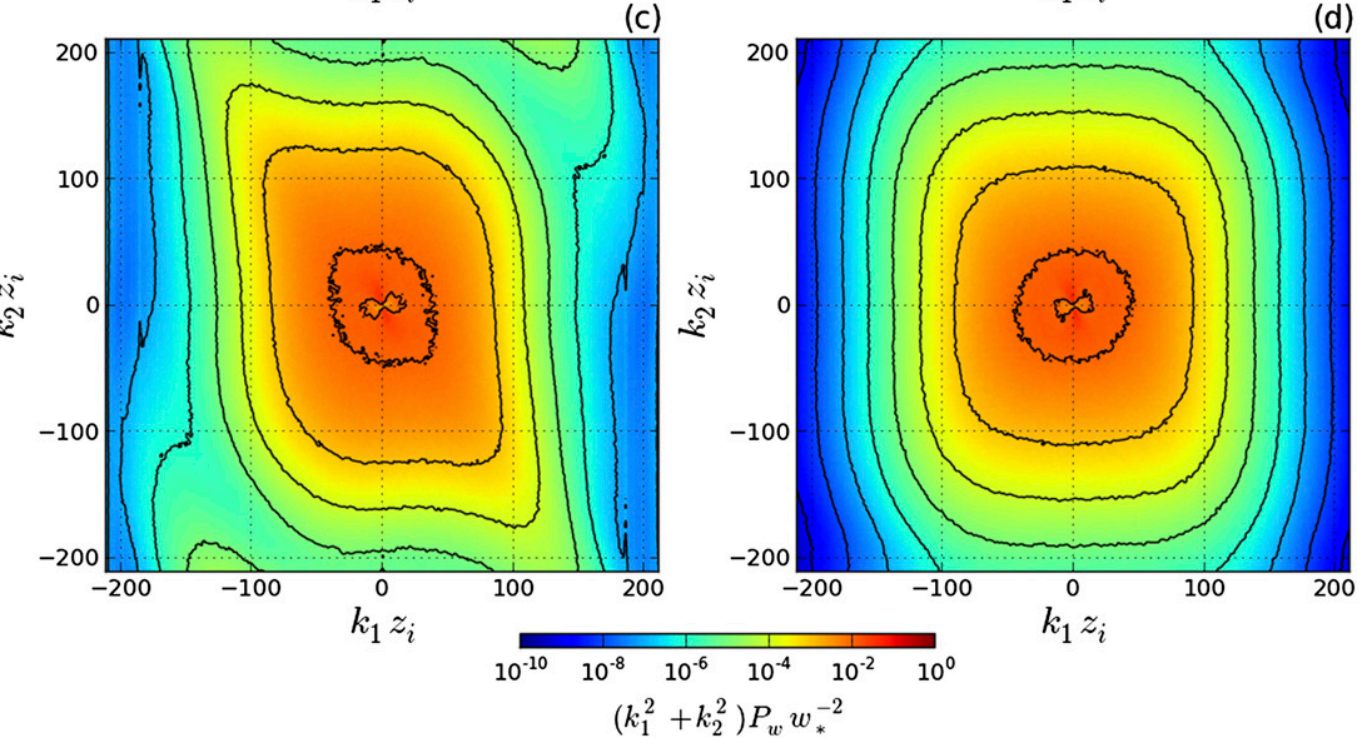

(d)

FIG. 7. As in Fig. 6, but for the $w$ component of the velocity.

one-dimensional (1D) spectral density of the $u$ component (see Fig. 4).

Normalized 2D spectra of the $w$-velocity component are shown in Fig. 7. Again, spectra in Figs. 7a and 7b (Figs. 7c,d) are from the shear-free (sheared) CBL simulations. Figures $7 \mathrm{a}$ and $7 \mathrm{c}$ (Figs. 7b,d) illustrate spectra calculated from OU-LES (WRF-LES) data. In this case, both $x\left(k_{1}\right)$ and $y\left(k_{2}\right)$ directions are transverse directions in relation to the $w$ component of velocity. Immediately evident in this case is the practically circular geometry of the spectral contours associated with the approximate turbulence isotropy within the inertial subranges of the spectra. This feature is again consistent with predictions of the turbulence theory. Indeed, since both the $x$ and $y$ directions are transverse directions in this case, the ratios of the inertial-subrange spectral densities of $w$ along the corresponding wavenumber coordinates should approximately equal unity (it should exactly be unity for perfectly isotropic turbulence). One may also notice that spectra from WRF-LES are again more squeezed toward larger scales (smaller wavenumbers) and show a sharper dropoff at large wavenumbers than do the OU-LES spectra, although the effect is less pronounced than for $u$-component spectra in Fig. 4. This behavior matches that which was previously observed in the one-dimensional $w$-component spectral density.

On the other hand, 2D $w$ spectra for the shear-driven CBL (Figs. 7c,d) are noticeably stretched in the $k_{2}$ direction. This would be consistent with the flow field in which more energy of the vertical velocity component on smaller scales is concentrated in the across-roll 
(roughly along $y$ ) direction. This feature also underscores the relatively small variability of the vertical velocity and fast decay of kinetic energy of vertical motion along the rolls. Similarly to the $u$-component 2D spectral pattern (Fig. 7), the OU-LES $w$ spectral contours at large-magnitude wavenumbers appear noticeably more stretched in the cross-flow direction than the WRF-LES spectral contours that look relatively directionally uniform. Like in the case of horizontal velocity spectra, these stretched portions in the OU-LES $w$ spectrum point to the enhanced energy of vertical velocity fluctuations due to the apparent shear-induced effects retained in the OU-LES flow on small scales.

Based on consideration of the 1D spectral densities, we suggested in section $3 b$ that the WRF model has a lower effective resolution than did OU-LES and that the potential cause of this feature is the excessive damping of large-wavenumber (small scale) turbulent velocity fluctuations by the numerical solver of the WRF model. The presented 2D spectra provide additional insights into the directional and scale-dependent properties of the simulated flow that result from such model performance. As expected, the turbulence appears nearly isotropic over horizontal planes in the shear-free CBL. The corresponding 2D spectra from WRF-LES point to enhanced numerical damping of turbulence at large wavenumbers. On the other hand, there is evidence of larger energy values within small-wavenumber ranges of the WRF-LES spectra. Enhanced numerical damping is also seen in the 2D sheared CBL spectra from WRFLES, where turbulence appears to be mostly affected in the across mean-wind (across roll) direction.

While small scales of turbulent motion within the shear-free CBL are often not considered to be as meteorologically relevant as they are, their potential influence on the turbulent motions within a broader range of turbulence scales may have farther reaching consequences. It may be hypothesized that the combined effect of various numerical filters and implicit damping associated with the employed horizontal advection scheme in the WRF model is an apparent cause of the reported spectral behavior on small scales. The observed energy enhancement at large scales may be a result of energy redistribution between scales by the employed numerical scheme. In physical terms, turbulence on smaller scales drains energy from larger resolved scale turbulence motions (Domaradzki et al. 1993). If the smallscale turbulent velocity fluctuations are overly damped, the turbulence energy cascade on larger scales is also affected.

Potential effects of the numerical filters employed in the WRF model on the turbulence simulations were also considered (Wicker and Skamarock 2002; Takemi and Rotunno 2003; Klemp et al. 2007). Six additional simulations were conducted (three for shear-free and three for sheared CBL cases). Each simulation was run with a uniform grid spacing of $80 \mathrm{~m}$. One of the three time-splitting filters was disengaged in each run. The effect of each filter resulted in minimal (of the order $<10 \%)$ and inconsistent spectral differences across scales (for the sake of brevity, these data are not shown). For instance, turning off the vertically integrated horizontal divergence damping brought the spectral distribution from WRF-LES closer to that from OU-LES, while the disconnect of three-dimensional divergence brought them farther apart. It is clear that the effects of filters alone cannot account for the observed differences between the OU-LES and WRF-LES spectral densities. This points to the underlying model numerics as the main source of the discrepancies between spectra originating from the two codes. The implication is that higher-order numerics may affect the entire turbulence spectrum. It is also important to note that disabling the time-splitting filters is unwise. The lack of three-dimensional divergence damping is known to generate excessive noise, while the lack of external-mode damping could create problems during the initialization stage of the simulation (J. Dudhia 2013, personal communication).

\section{d. Turbulence statistics}

To glean additional insight into the CBL flow properties revealed by the velocity spectra, we now take a look at some basic velocity-field statistics. Histograms of velocity components during the final hour of the simulation window are demonstrated in Fig. 8. The top panels in Fig. 8 present perturbations of the $u, v$, and $w$, respectively, velocity components for the shear-free CBL case. The bottom panels in Fig. 8 display histograms of the velocity perturbations for the sheared CBL case.

For a finite statistical sample $\left\{x_{i}\right\}$ of size $N$ (where $i=$ $1, \ldots, N)$, the $n$th statistical moment is defined as

$$
\mu_{n}=\frac{1}{N} \sum_{i=1}^{N}\left(x_{i}-\bar{x}\right)^{n},
$$

where $\bar{x}$ is the population mean. Using this definition, one can subsequently define other commonly used statistics that include standard deviation, skewness, and kurtosis.

Standard deviation is the square root of the $\left\{x_{i}\right\}$ variance represented by the second moment $\mu_{2}$ :

$$
\sigma=\sqrt{\mu_{2}} .
$$



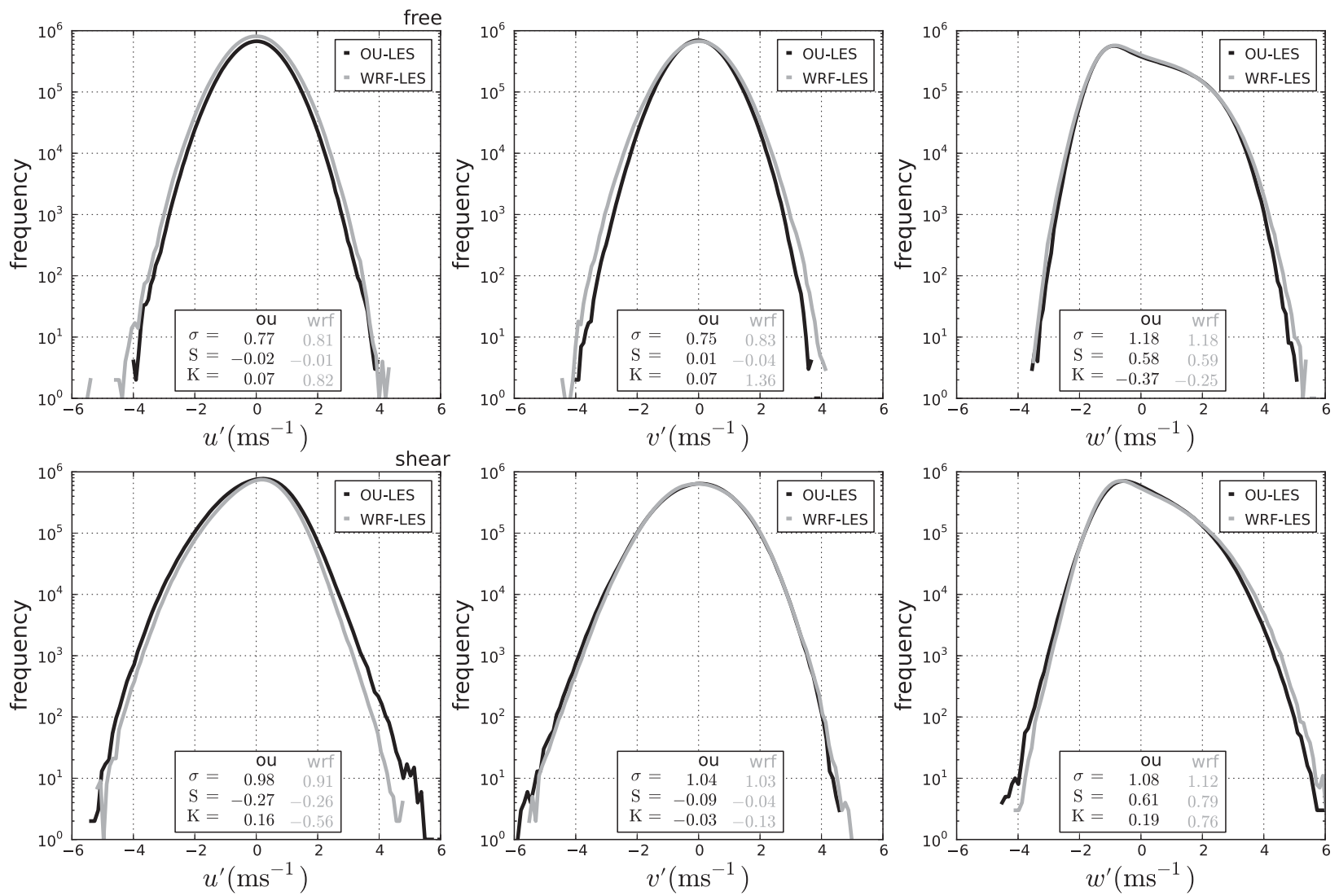

FIG. 8. Histograms of the resolved velocity components for the final hour of the simulation window at $z / z_{i}=0.25$ for the (top) shear-free and (bottom) sheared CBL cases. Values of $\sigma$ are in meters per second.

Skewness characterizes the asymmetry of the $\left\{x_{i}\right\}$ distribution about the mean and is defined as

$$
S=\mu_{3} / \sigma^{3}
$$

A negative (positive) value for skewness corresponds to a longer left (right) tail on the distribution curve, with the bulk of the values being concentrated on the right (left).

Kurtosis is a measure of the peakedness of the $\left\{x_{i}\right\}$ distribution about the mean. It is given by

$$
K=\frac{\mu_{4}}{\sigma^{4}}-3
$$

where the subtraction of 3 is meant to set $K$ to zero for the normal distribution. In this standardized form, $K$ is often referred to as excess kurtosis. It is important to keep this normalization in mind when comparing the reported kurtosis values. Large (small) values of kurtosis mean that more (less) variance originates from infrequent extreme deviations from the mean. In the case of $\left\{x_{i}\right\}$ being a sample of wind velocity values, these deviations can be thought of as wind gusts. Distributions with a positive (negative) value of kurtosis are referred to as leptokurtic (platykurtic). They have shapes characterized by a higher (lower) peak around the mean and thicker (thinner) tails as compared to the normal distribution. Values of standard deviation $\sigma$, skewness $S$, and kurtosis $K$ corresponding to the particular velocity distribution are given in the panels of Fig. 8.

In the shear-free CBL, the horizontal velocity from WRF-LES has a larger standard deviation than its counterpart from OU-LES, similar skewness, and much larger positive kurtosis. The skewness values are rather small for both simulations, but still indicate that velocity distributions are slightly negatively skewed. The disparity in kurtosis reveals that OU-LES nearly evenly distributes the horizontal velocity about the mean, with departures arising from frequent, although modest in magnitude, deviations from the mean. Oppositely, the leptokurtic nature of the horizontal velocity distribution from WRF-LES is associated with a more acute distribution peak near the mean with longer tails as compared, for example, to the normal distribution. This means that data are largely concentrated around the mean as a result of reduced variations within the velocity field. While the 
overall variability across the horizontal plane is smaller in the WRF-LES field as compared with the OU-LES velocity distribution, WRF-LES velocity variations are more likely to originate from extreme deviations from the mean. In other words, wind velocity in WRF-LES exhibits an increased gustiness. Such behavior is consistent with the velocity-field features demonstrated in Fig. 2 and could also be linked to the energy accumulation on large scales pointed out in the spectra shown in Fig. 4. The wind gustiness is even more pronounced in the $v$-component velocity from WRF-LES, whose almost zero-skewed distribution displays longer tails in both positive and negative velocity ranges.

The standard deviations of the vertical velocity for the shear-free case are practically identical for both simulation techniques. Also close are the essentially positive skewness values from both simulations. Positive skewness is a signature feature of the $w$ field in a shear-free CBL (LeMone 1990). The vertical velocity field from OU-LES, however, has a slightly larger negative kurtosis. The more platykurtic nature of the OU-LES velocity distribution means a flatter peak around the mean as compared to the normal distribution and to the WRFLES data. Apparently, WRF-LES again produces an overall less variable velocity field than OU-LES, with more values centered around the mean and a few deviating strongly from the mean, which results in a longer distribution tail. Although in the case of the $w$ distribution, this effect of the larger kurtosis of WRF-LES deviations is less pronounced than in the cases of the horizontal velocity components. Such behavior is in general agreement with structural features of the flow observed in Fig. 3 and revealed by velocity spectra in Figs. 5 and 7.

In the sheared CBL, horizontal velocity distributions from OU-LES feature a higher standard deviation and similar moderately negative skewness in comparison with WRF-LES data. OU-LES data also display a small positive kurtosis versus a larger negative kurtosis of the $u$ distribution from WRF-LES. Accordingly, horizontal velocity values from WRF-LES are less concentrated around the mean than are the data from OU-LES (as also reflected in Fig. 2). The variability of the OU-LES field is more due to infrequent and extreme departures from the mean. Meanwhile, the vertical velocity in the sheared CBL case from WRF-LES has a larger standard deviation, a larger positive skewness, and larger positive kurtosis. This means that, as compared with OU-LES output, the WRF-LES $w$ data have a narrower distribution peak near the origin. However, infrequent and intense departures from the mean in the WRF-LES $w$ distribution are more characteristic of the positive velocity fluctuations (associated with updrafts), which is additionally testified to by the smaller skewness value of the WRF-LES $w$ sample. While the degree of variability differs between the WRF-LES horizontal and vertical velocity fields in the sheared CBL case, the velocity histograms are generally indicative of larger flow gustiness in the OU-LES data. These observation agrees with previously discussed elongated and intense roll-like structures reproduced by WRF-LES, whereas the OULES flow pattern displays more variability in the crossroll direction (Figs. 2 and 3).

Figures 9a-c (Figs. 9d-f) illustrate vertical profiles of velocity-component variances in the shear-free (sheared) CBL case. For both horizontal and vertical velocities in the shear-free case, WRF-LES exhibits larger variances throughout most of the layer and these are consistent with the velocity distribution at $z / z_{i}=0.25$ depicted in Fig. 8, where the WRF-LES velocity field shows a tendency toward more contrasted structural features. In the sheared CBL case, however, OU-LES produces larger variances for the $u$ component of the wind, with the variances of the $v$ and $w$ components at $z / z_{i}=0.25$ being nearly identical between the two simulations. Again, the discussed behavior of the velocitycomponent variances is in general agreement with the horizontal slices of the velocity field (Figs. 2 and 3) and the associated velocity distribution Fig. 8 in the sheared CBL. Spectra of the WRF-LES velocity fluctuations also point to the decreased variability of the velocitycomponent fields on smaller scales in the simulated sheared CBL.

Vertical profiles of TKE from both simulations are shown in Fig. 10. As expected from the previously discussed velocity distributions and profiles of variances, WRF-LES produces greater energy as compared with OU-LES throughout the major portion of the shear-free CBL. This appears to be in agreement with the earlier finding that WRF-LES is attributing more energy to larger scales of motion in this case (Figs. 4 and 5). Such an accumulation of energy on larger scales is also made evident by the broader, less organized large-scale structures in the shear-free CBL reproduced with WRF-LES (see Fig. 3). Oppositely, in the sheared CBL, OU-LES produces larger TKE values than WRF-LES does, particularly in the near-surface portion of CBL, where both codes consistently predict the turbulence enhancement due to the shear generation of TKE. The greater enhancement of TKE in the lower portion of the CBL by the OU-LES code is in line with the velocity distributions and variances for this CBL case (Figs. 8 and 9). Both codes predict the dominance of the resolved TKE over the subgrid TKE component throughout the whole CBL. In the sheared CBL case, one may see the relative increase of the subgrid TKE contribution within the 

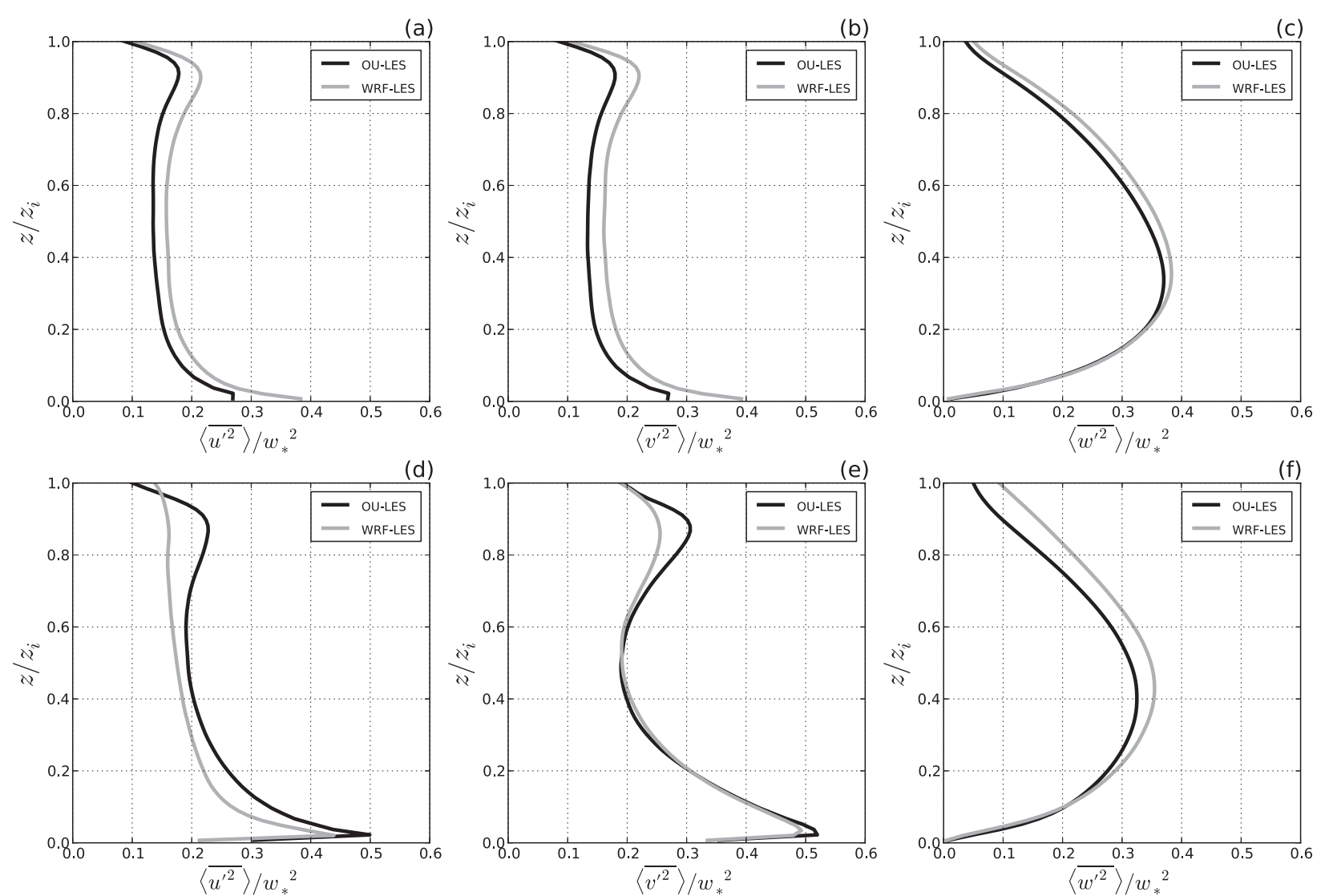

FIG. 9. Normalized hourly mean variances of the resolved velocity components for the final hour of the simulation window for the (a)-(c) shear-free and (d)-(f) sheared CBL cases.

strongly sheared flow region at $z / z_{i}<0.25$. For this CBL case, the structure of the TKE profile may be used to explain some features of the spectral density behavior. Reduced TKE in the lower portion of the simulated flow from WRF-LES is apparently associated with relatively less mixing of momentum and relatively stronger nearsurface shear in the WRF-LES case. The profiles of the $x$ component of the kinematic momentum flux shown in Fig. 11 indicate that the vertical transport of momentum in the lower region of the WRF-LES CBL is comparatively less than in the OU-LES CBL. The wind direction was consistent between codes (the corresponding data are not shown). The associated enhanced mean nearsurface shear on the WRF-LES flow case is clearly seen in Fig. 12. Such a combination of stronger shear and weaker TKE points to the fact that kinetic energy on smaller scales of motion, characteristic of the nearsurface portion of the sheared CBL, is not sufficiently resolved in WRF-LES. This stronger shear and the greater damping of small-scales motions, apparently associated with it, result in more coherent roll-like structures in the WRF-LES CBL and the faster decay of the corresponding velocity spectra toward large wavenumbers.

\section{Summary and discussion}

Large-eddy simulations of idealized turbulent flow in the atmospheric convective boundary layer were conducted for two differing CBL flow types: shear-free and shear-driven. A conventional large-eddy-simulation code, OU-LES, was employed in conjunction with WRFLES, the WRF model that was run in LES mode. Differences in overall CBL flow behavior for both codes were found to be minor across disparate uniform grid spacings of $20-80 \mathrm{~m}$. Subsequently, simulation results were analyzed in detail only for the finest-scale $(20 \mathrm{~m})$ runs.

Sample slices of instantaneous velocity fields produced by OU-LES and WRF-LES were exposed for visual inspection. While a cursory glance might have yielded the feeling that simulated fields were largely the same, a closer examination determined that subtle differences did exist. Accordingly, a deeper investigation of the underlying flow structure features was conducted to ascertain possible reasons for those differences. Spectral analysis was applied as the principal method to evaluate velocity fields produced by each simulation approach. 

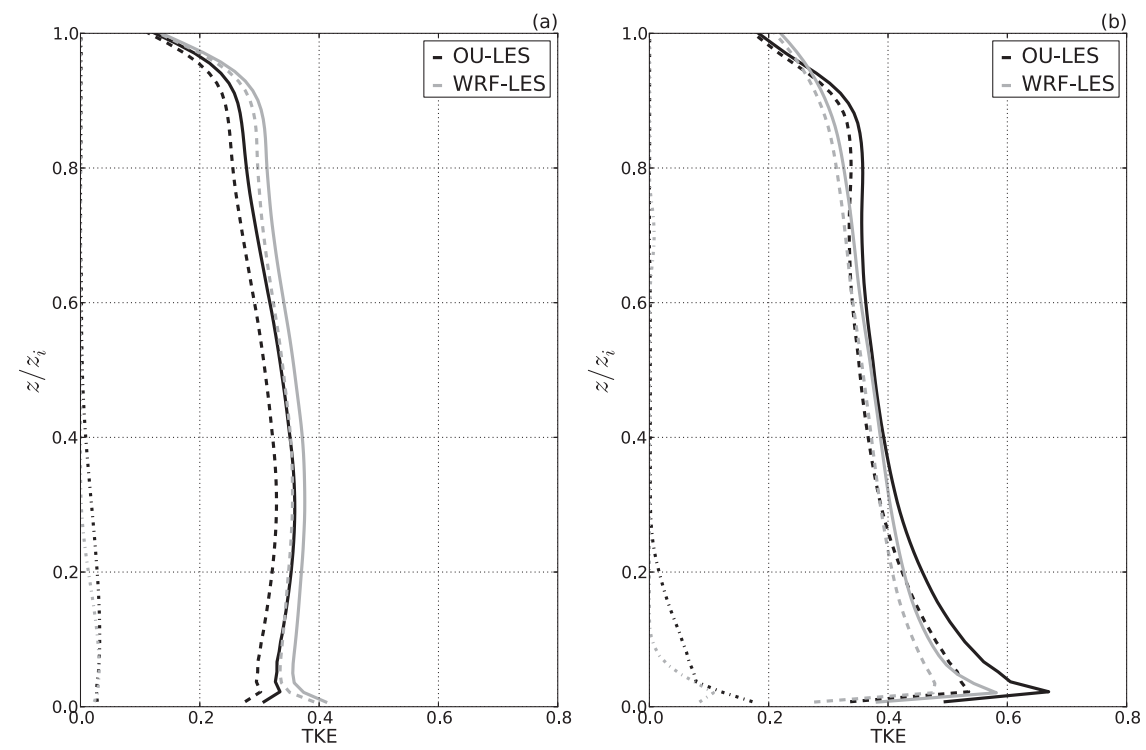

FIG. 10. Normalized hourly mean TKE for the final hour of the simulation window. Dashed lines represent resolved TKE, $\left.0.5\left(\left\langle\overline{\left\langle u^{\prime 2}\right.}\right\rangle+\overline{\left\langle v^{\prime 2}\right.}\right\rangle+\left\langle\overline{{w^{\prime 2}}^{2}}\right\rangle\right) / w_{*}^{2}$; dashed-dotted lines represent subgrid TKE; and solid lines represent total TKE. Shown are the (a) shear-free and (b) sheardriven cases.

Other statistics of the simulated velocity fields, including velocity-component histograms, higher-order moments (variance, covariance, skewness, and kurtosis), and turbulence kinetic energy have been examined complementary to $1 \mathrm{D}$ and $2 \mathrm{D}$ velocity spectra.

In the shear-free CBL case, one-dimensional $u$ component spectral density indicated that the WRFLES velocity field contains more energy at larger scales and the deviation from the expected spectral slope occurs earlier (at smaller wavenumbers) and happens at a higher rate than in the velocity field from OU-LES . For the $w$-component spectral density, the same behavior was observed, although the energy values at smaller wavenumbers were more comparable between the OU-LES and WRF-LES spectra. Two-dimensional spectra illustrated that both simulation methods produce inertial-subrange energy spectra in general agreement with turbulence theory predictions. However, 2D WRFLES spectra revealed the enhanced (apparently, numerical) damping of small-scale turbulent motions, which resulted in a coarser effective resolution of the simulation.

In the sheared CBL spectra, the energy enhancement on large scales was generally less pronounced. The energy drop in the 1D $u$-component spectrum from WRFLES happened relatively early compared to the OU-LES spectrum, although the rate of energy decline with increasing wavenumber was about the same. The similar behavior was found in the 1D $w$ spectrum in the $y$ direction, but the OU-LES and WRF-LES 1D $w$ spectra in the $x$ direction matched each other very closely. The two-dimensional spectra for this CBL case highlighted differences between the two codes in predicting energy distributions associated with the presence of shear. The OU-LES code produced 2D spectra with stretched

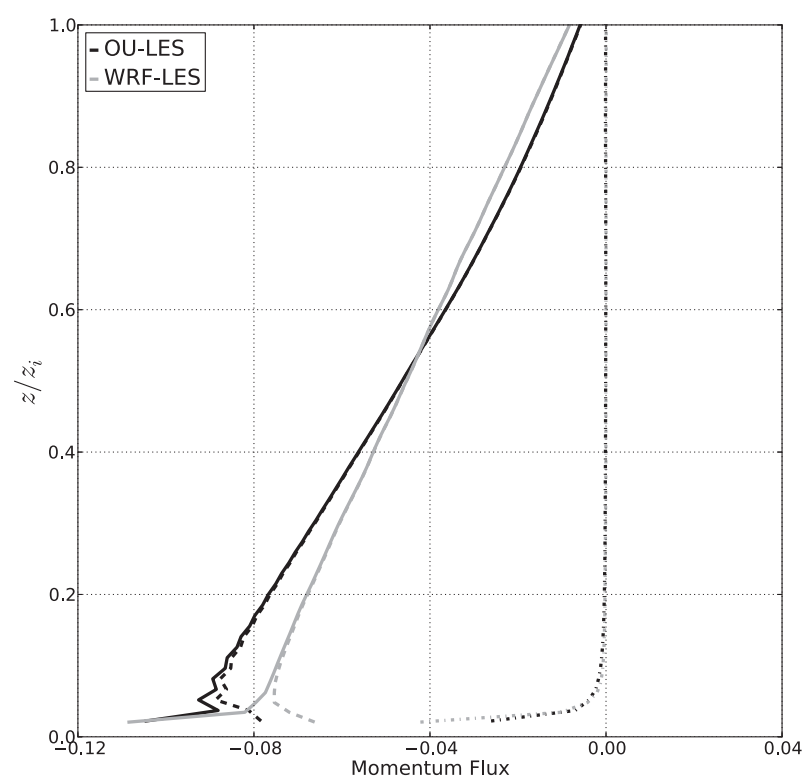

FIG. 11. Normalized $x$ component of the vertical turbulent momentum flux for the final hour of the sheared CBL simulation. Dashed lines represent resolved flux, $\left\langle\overline{w^{\prime} u^{\prime}}\right\rangle / w_{*}^{2}$; dashed-dotted lines represent subgrid flux; and solid lines represent the total flux. 


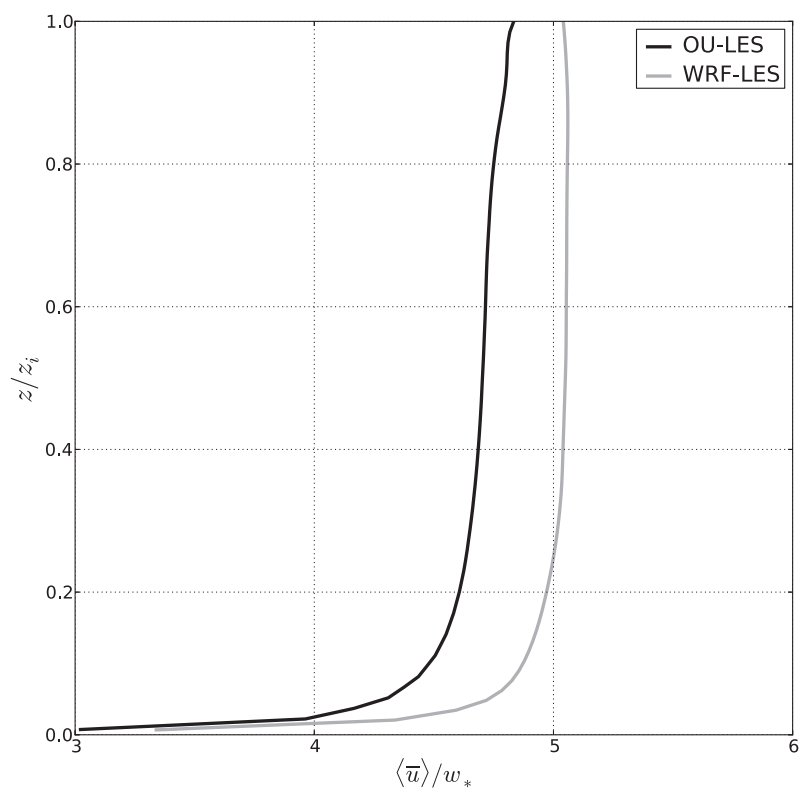

FIG. 12. Normalized mean $u$-component velocity profile for the final hour of the simulation window.

contours at large wavenumber magnitudes (small scales) - a feature that was absent in the WRF-LES spectra. In terms of the physical structure of the velocity field, this is an indication of OU-LES reproducing CBL flow with larger variability on small scales in the crosswind direction. Conversely, the WRF-LES code did not reproduce these small-scale anisotropic features of velocity fields in the sheared CBL.

Additional statistical analyses were conducted to further aid our understanding of the spectral data. Histograms of the $u$-velocity component from the shearfree CBL indicated that WRF-LES generated velocity distributions with greater positive kurtosis than that of the distributions from OU-LES. WRF-LES also produced a $u$ field with smaller negative kurtosis in the sheared CBL, thus once again pointing to a less-variable flow field with closer concentration around the mean. These features, which are consistent with the large-scale kinetic energy concentration and sharper drop toward small scales in the WRF-LES velocity fields, provided support for the results of the spectral analyses of velocity fields. In the sheared CBL case, distributions of the $u$-velocity component in OU-LES pointed to horizontal velocity fields more variable and less concentrated around the mean than the ones resulting from WRF-LES. For vertical velocity $w$ in the sheared CBL, the OU-LES data produced smaller positive kurtosis values as compared to the WRF-LES data. Subsequently, WRF-LES velocity fields contained more elongated, broader, and intense velocity structures associated with roll-like motions. Conversely, OU-LES fields featured more variability across the mean wind.

Vertical profiles of velocity variance and turbulence kinetic energy were found to be consistent with velocity spectra and histogram revelations. The corresponding profiles confirmed the relatively larger values of kinetic energy on larger scales in the shear-free CBL velocity field from WRF-LES. On the other hand, in the sheared CBL case, turbulence kinetic energy of the WRF-LES velocity fields was inhibited compared with the OU-LES data. The enhanced near-surface mean shear in WRFLES was associated with the relatively weak vertical momentum flux, indicating more effective mixing of momentum near the surface in the OU-LES flow field. Subsequently, WRF-LES produced a mean wind profile structurally similar to its OU-LES counterpart but stronger in magnitude throughout the whole CBL depth.

A question arises as to why the flow features pointed out were specifically characteristic of WRF-LES output since the flow setups for both simulation approaches were basically the same. Given that one main difference between the approaches lies in the way the simulated fluid is treated (as compressible in the WRF-LES case versus incompressible in the OU-LES case), one could argue that a set of filters in the WRF model's timesplitting procedure employed to mitigate compressibility effects may at least be partially responsible for such behavior in the model solutions. The following three filters are implemented. First, the temporal averaging in the time-splitting procedure for the vertical momentum and geopotential equations is slightly centered forward to enhance damping of short-wave disturbances in the vertical. Second, a modified pressure, employed in the horizontal momentum equations, is designed as a filter to specifically damp three-dimensional divergence of the velocity field. Finally, a filter-type term is added to the horizontal momentum equations that acts to damp the vertical integral of the horizontal divergence.

While Skamarock and Klemp (1992) suggest that these filters should only influence the smallest scales, concessions are made in their work that larger scales of motion could also be affected. The filters are also mentioned as a possible source of error in Skamarock (2004), but with the justification that the impacted scales were not of "meteorological interest." While that might be true for mesoscale applications of the WRF model, it is apparently not the case for the WRF model being applied in the LES mode, where the excessive damping of small-scale motions appears to be rather persistent. Such damping can potentially have a negative effect on, for example, air pollution applications where the dispersive role of small-scale motions may be important, or in wave 
propagation applications where the variability of flow fields on small scales in terms of structure functions would not be appropriately quantified. The effect of timesplitting filters on spectra from WRF-LES were inconsistent across scales and minimal in magnitude. The conclusion was reached that filter effects alone could not explain the discrepancies between the OU-LES and WRF-LES spectra and that the observed differences in the energy distribution over the turbulence spectra are primarily caused by differences in model numerics.

Why is energy attribution to large-scale motions particularly characteristic of the WRF-LES predictions for the shear-free CBL flow? A potential reason for such behavior may be the usage of the fifth-order advection scheme in WRF-LES. As indicated in Wicker and Skamarock (2002), an odd-order advection scheme would be equivalent to the next even-order advection approximation with an added damping term proportional to the flow speed. In the case of a strong mean wind, the implicit damping associated with horizontal advection would be relatively strong and would surpass the effect of the time-split numerical filters that target only smallscale modes. Accordingly, motions in a broad range of scales, not only small-scale motions, would be damped in the sheared CBL. This gives further credibility to the theory that model numerics are of vital importance to the resulting spectral distributions.

While the reasons for pronounced numerical damping on small scales of motion and the energy attribution to the large scale in the WRF-LES certainly require further study, their impact on model predictions should be taken into account when applying the WRF model for realistic atmospheric conditions. In such applications, higher-order advection schemes are desirable given the push for increased spatial accuracy. The associated numerical damping, however, could be a complication in this case. Additionally, specified lateral boundaries must be used in practical WRF model applications in place of periodic lateral boundaries. The inclusion of specified lateral boundaries is known to introduce spurious eddydeficient areas that may extend over a large portion of the inflow region of the simulation domain (Moeng et al. 2007). These problems can become more severe because of the poor representation of interactions between subgrid-scale motions, often insufficiently treated by commonly employed subgrid closure schemes, and three-dimensional resolved LES flow fields. Another concern emerging from our study is related to the reduction of the effective resolution of WRF-LES by the combined effects of model numerics and filters employed in the WRF model's time-splitting procedure. Under such conditions, the reliability of the reproduction of scales of motion important for particular applications may be called into question, and the overall applicability of WRF-LES in certain practical applications may be limited.

If a particular study primarily hinges on accurate description of the energy propagation, then certainly the use of higher-order advection schemes makes sense. However, the results of this study indicate that such schemes may distribute energy over turbulence scales in a nonrealistic manner. To address some of these issues, one may also consider applying different subgrid-scale (SGS) models. If an SGS model adequately represents the energy cascade on small-scale motions, then presumably the overall energy distribution over the spectrum will improve. Prospective SGS models include the nonlinear backscatter and anisotropy model (Mirocha et al. 2010), the Lagrangian-averaged scale-dependent dynamic model (Bou-Zeid et al. 2005), and the dynamic reconstruction model (Chow et al. 2005). Another approach toward describing the turbulent boundary layer flow within realistic atmospheric environments could be based on nudging a conventional LES with mean flow fields from larger-scale model output (e.g., from the mesoscale WRF model output). In such a model configuration, the issue of eddy-deficient flow regions is largely eliminated. This particular downscaling technique has been proven successful in OU-LES applications to realistic atmospheric environments (Botnick and Fedorovich 2008; Gibbs et al. 2011).

Acknowledgments. The authors gratefully acknowledge support from the Netherlands Organisation for Applied Scientific Research (TNO) and the National Science Foundation within Research Grant ATM-1016153.

\section{REFERENCES}

Botnick, A. M., and E. Fedorovich, 2008: Large eddy simulation of atmospheric convective boundary layer with realistic environmental forcings. Quality and Reliability of Large-Eddy Simulations, J. Meyers, B. Geurts, and P. Sagaut, Eds., Springer, 193-204.

Bou-Zeid, E., C. Meneveau, and M. Parlange, 2005: A scaledependent Lagrangian dynamic model for large eddy simulation of complex turbulent flows. Phys. Fluids, 17, 025105 , doi:10.1063/1.1839152.

Chow, F. K., R. L. Street, M. Xue, and J. H. Ferziger, 2005: Explicit filtering and reconstruction turbulence modeling for largeeddy simulation of neutral boundary layer flow. J. Atmos. Sci., 62, 2058-2077.

Deardorff, J. W., 1970: Convective velocity and temperature scales for the unstable planetary boundary layer and for Rayleigh convection. J. Atmos. Sci., 27, 1211-1213.

_ 1980 : Stratocumulus-capped mixed layers derived from a three-dimensional model. Bound.-Layer Meteor., 18, 495-527.

Domaradzki, J. A., W. Liu, and M. E. Brachet, 1993: An analysis of subgrid-scale interactions in numerically simulated isotropic turbulence. Phys. Fluids A, 5, 1747-1759. 
Dyer, A. J., and B. B. Hicks, 1970: Flux-gradient relationships in the constant flux layer. Quart. J. Roy. Meteor. Soc., 96, 715-721.

Fedorovich, E., F. T. M. Nieuwstadt, and R. Kaiser, 2001: Numerical and laboratory study of horizontally evolving convective boundary layer. Part I: Transition regimes and development of the mixed layer. J. Atmos. Sci., 58, 70-86.

, R. Conzemius, and D. Mironov, 2004a: Convective entrainment into a shear-free linearly stratified atmosphere: Bulk models reevaluated through large eddy simulations. J. Atmos. Sci., 61, 281-295.

, and Coauthors, 2004b: Entrainment into sheared convective boundary layers as predicted by different large eddy simulation codes. Preprints, 16th Symp. on Boundary Layers and Turbulence, Portland, ME, Amer. Meteor. Soc., P4.7. [Available online at https://ams.confex.com/ams/pdfpapers/78656.pdf.]

Gibbs, J. A., E. Fedorovich, and A. M. J. van Eijk, 2011: Evaluating Weather Research and Forecasting (WRF) model predictions of turbulent flow parameters in a dry convective boundary layer. J. Appl. Meteor. Climatol., 50, 2429-2444.

Kaiser, R., and E. Fedorovich, 1998: Turbulence spectra and dissipation rates in a wind tunnel model of the atmospheric convective boundary layer. J. Atmos. Sci., 55, 580-594.

Kelly, M., and J. C. Wyngaard, 2006: Two-dimensional spectra in the atmospheric boundary layer. J. Atmos. Sci., 63, 3066-3070.

Klemp, J. B., and W. Skamarock, 2004: Model numerics for convective-storm simulation. Atmospheric Turbulence and Mesoscale Meteorology, E. Fedorovich, R. Rotunno, and B. Stevens, Eds., Cambridge University Press, 117-138.

- — - and J. Dudhia, 2007: Conservative split-explicit time integration methods for the compressible nonhydrostatic equations. Mon. Wea. Rev., 135, 2897-2913.

LeMone, M. A., 1990: Some observations of vertical velocity skewness in the planetary boundary layer. J. Atmos. Sci., 47, 1163-1169.

Mirocha, J. D., J. K. Lundquist, and B. Kosović, 2010: Implementation of a nonlinear subfilter turbulence stress model for large-eddy simulation in the Advanced Research WRF model. Mon. Wea. Rev., 138, 4212-4228.
Moeng, C.-H., and P. P. Sullivan, 1994: A comparison of shear- and buoyancy-driven planetary boundary layer flows. J. Atmos. Sci., 51, 999-1022.

_ - J. Dudhia, J. Klemp, and P. Sullivan, 2007: Examining twoway grid nesting for large eddy simulation of the PBL using the WRF model. Mon. Wea. Rev., 135, 2295-2311.

Monin, A. S., and A. M. Obukhov, 1954: Basic laws of turbulent mixing in the surface layer of the atmosphere. Contrib. Geophys. Inst. Acad. Sci., USSR, 151, 163-187.

Nieuwstadt, F. T. M., 1990: Direct and large-eddy simulation of free convection. Proc. Ninth Int. Heat Transfer Conf., Jerusalem, Israel, American Society of Mechanical Engineers, 37-47.

Pope, S. B., 2000: Turbulent Flows. Cambridge University Press, $771 \mathrm{pp}$.

Skamarock, W. C., 2004: Evaluating mesoscale NWP models using kinetic energy spectra. Mon. Wea. Rev., 132, 3019-3032.

_ , and J. B. Klemp, 1992: The stability of time-split numerical methods for the hydrostatic and the nonhydrostatic elastic equations. Mon. Wea. Rev., 120, 2109-2127.

,-- J. Dudhia, D. O. Gill, D. M. Barker, W. Wang, and J. G. Powers, 2008: A description of the Advanced Research WRF version 3. NCAR Tech. Note NCAR/TN-475+STR, 113 pp. [Available online at www.mmm.ucar.edu/wrf/users/docs/ arw_v3.pdf.]

Sullivan, P. P., J. C. McWilliams, and C.-H. Moeng, 1996: A grid nesting method for large-eddy simulation of planetary boundary-layer flows. Bound.-Layer Meteor., 80, 167-202.

Takemi, T., and R. Rotunno, 2003: The effects of subgrid model mixing and numerical filtering in simulations of mesoscale cloud systems. Mon. Wea. Rev., 131, 2085-2101.

Tennekes, H., and J. L. Lumley, 1972: A First Course in Turbulence. The MIT Press, 300 pp.

Wicker, L. J., and W. C. Skamarock, 1998: A time-splitting scheme for the elastic equations incorporating second-order RungeKutta time differencing. Mon. Wea. Rev., 126, 1992-1999.

_ , and —, 2002: Time-splitting methods for elastic models using forward time schemes. Mon. Wea. Rev., 130, 2088-2097. 